This is the authors' copy of the unpublished version of this manuscript, which is currently under review.

Please cite as:

Masapollo, M., Smith, D.J, \& Guenther, F.H. (under review). Dissociation between phonological working memory structures and motor programming units in speech motorsequence learning.

\title{
Dissociation between phonological working memory structures and motor programming units during speech motor-sequence learning
}

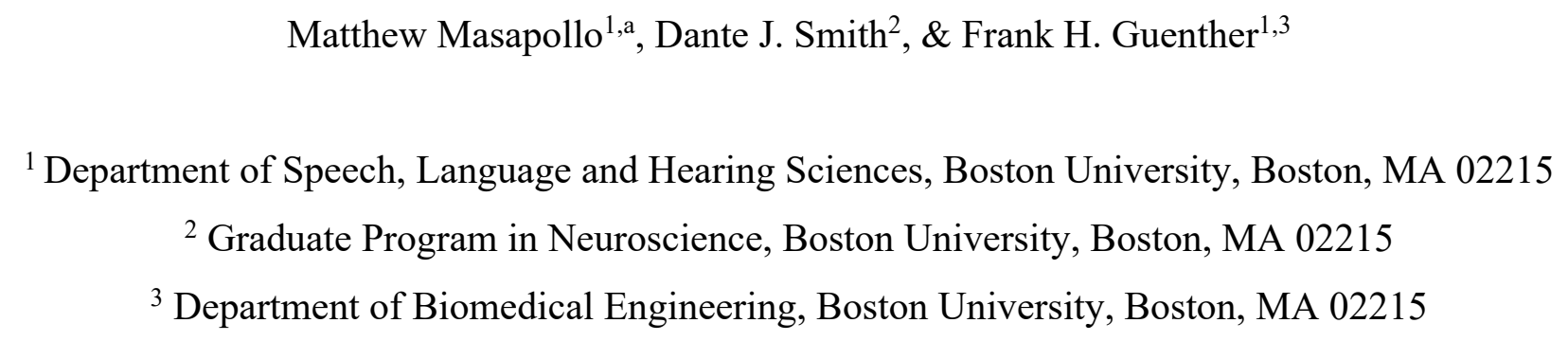

To whom correspondence should be addressed. Email: mmasapollo@phhp.ufl.edu .

Funding: The research reported in this publication was supported by a grant from the National Institutes of Health (R01 DC007683; F.H. Guenther, PI). The content is solely the responsibility of the authors and does not necessarily represent the official views of the National Institutes of

Health. Matthew Masapollo was also supported by research funds from the University of Florida during the preparation of this manuscript.

\footnotetext{
a Current address: Department of Speech, Language, and Hearing Sciences, University of Florida, Gainesville, FL 32610
} 
Abstract

Purpose: This study investigated the nature of phonological working memory (PWM)

23 structures and speech motor programming units by examining how performance gains from

24 practicing non-native phoneme sequences generalize to novel sequences that overlap to varying

25 degrees with the practiced sequences.

26 Method: CCVCC words were constructed using consonant clusters that violated English

27 phonological constraints, thus making them difficult to produce initially. After practicing a subset

28 of the words over two consecutive days, participants were tested on the production of several types

29 of word pairs, including novel words containing unpracticed clusters, practiced cluster words

30 containing consonant clusters that were practiced but in different words from the test words, and

31 fully learned words that were practiced in their entirety.

32 Results: Utterance duration improvements from practicing clusters in one syllabic context

33 fully transferred to novel words that included these clusters, while error rate improvements from

34 practicing clusters in one syllabic context only partially generalized to new syllables utilizing these

35 practiced clusters. Additionally, error rates for the first word in the pair (which depend primarily

36 on motor program structure) showed partial improvements for learned clusters regardless of

37 whether the cluster was practiced in the same part of the syllabic frame (onset or coda), whereas

38 error rates for the second word (which reflect both PWM and motor programming mechanisms)

39 were higher than even novel words if the cluster was practiced in the wrong syllable frame location,

40 presumably due to interference effects in PWM.

41 Conclusions: These results provide support for an onset-nucleus-coda syllabic frame

42 structure in PWM and a syllable-frame-independent representation of common phoneme sub-

43 sequences for motor programs. 
44 Keywords: speech motor control; phonetic encoding; serial ordering; motor-sequence learning; 45 working memory; motor programming 


\section{Introduction} motor programming units in speech motor-sequence learning

One of the fundamental issues in the field of speech production is how speakers map discrete phonological units (e.g., phonemes, syllables, words) onto properly timed vocal tract

52 articulations which lead to an audible, intelligible speech signal. Over the years, considerable

53 progress has been made in describing the nature of the complex mapping between cognitive

54 planning units and motor programs that activate the muscles of the vocal tract (for discussion, see

55 Guenther, 2016). Current understanding is mediated by psycholinguistic and neurobiological models designed to explicate the interaction of motor, sensory, linguistic, and cognitive skills in

57 shaping articulatory behavior (e.g., Dell, 1986; Shattuck-Hufnagel, 1979, 1983, 1986, 1992, 2015;

58 Levelt, 1989; MacNeilage, 1998; Levelt, Roelofs, \& Meyer, 1999; Cholin, Levelt, \& Schiller,

59 2006; Goldrick \& Blumstein, 2006; Guenther, Ghosh, \& Tourville, 2006; Bohland, Bullock \&

60 Guenther, 2010; Christiansen \& Chater, 2016; Hickok, 2017). However, as with most issues

61 concerning speech, there is still much more work to be done.

The issue we address in the current research concerns how speakers learn to rapidly coordinate and implement multiple serial vocal tract movements (or gestures) associated with the

64 consonants and vowels within a planned utterance. To date, the majority of research on speech

65 production has focused on elucidating the mechanisms and processes involved in executing 66 individual phonemes and simple syllables. Relatively little is known about the cognitive structures

67 and processes involved in planning and producing complex or extended sequences of speech 68 movements (see, e.g., Bohland \& Guenther, 2006; Bohland et al., 2010; Rong, Isenberg, Sun \&

69 Hickok, 2018). However, a viable theory of language and speech production must provide a 
70 detailed mechanistic account of this complex serial behavior, which is characteristic of natural 71 communicative speech.

72 Investigating how speakers learn and execute speech sequences is a critical activity in

73 developing models of speech production for multiple reasons. First, production planning of

74 phoneme sequences has inherent cognitive demands: the to-be-articulated segments must be

75 phonetically encoded, concatenated and temporarily stored in a verbal working memory repository

76 in such a way that preserves their serial order (see, e.g., Acheson \& MacDonald, 2009; Bohland et

77 al., 2010; Shattuck-Hufnagel, 2015; Guenther, 2016). Second, speech motor sequencing

78 necessitates fine-grained articulatory control. Kinematic analyses indicate that the implementation

79 of clusters of consonants places multiple, concurrent demands on the speech articulators, primary

80 the tongue, because the vocal tract gestures for neighboring segments are rapidly produced in

81 partially overlapping time frames (e.g., Browman \& Goldstein, 1988; Byrd, 1996; Tiede, Shattuck-

82 Hufnagel, Johnson, Ghosh, Matthies, Zandipour, \& Perkell, 2007). Third, the production of

83 consonant sequences, and, in particular, those with high articulatory complexity, present a

84 challenge for first and second language learners (e.g., Song, Demuth, Shattuck-Hufnagel \&

85 Ménard, 2013; Wilson, Davidson, \& Martin, 2014) as well as clinical populations with motor

86 speech disorders (e.g., Buchwald \& Miozzo, 2012; Buchwald, Gagnon, \& Miozzo, 2017), with

87 clusters often omitted or reduced. Taken together, these factors suggest the existence of rich

88 cognitive systems and processes for learning how to plan and execute sequences of phonological

89 units.

90 Throughout the years, numerous models and theories, such as those advanced by Lashley

91 (1951), Shattuck-Hufnagel (1979, 1983, 1992), Levelt et al. (1999), Bohland et al. (2010), and

92 Christiansen and Chater (2016), have commonly proposed that speakers produce frequently 
93 occurring sequences of phonological units by parsing them into larger units, or "chunks," at

94 various stages of the speech production planning process. At the phonological encoding stage,

95 where the planned utterance is mapped from morphemes onto syllables and phonemes, some have

96 proposed that such a chunking process would allow groups of individual phonemes to be selected,

97 concatenated, and stored as a cohesive cognitive planning unit in verbal working memory, which

98 would facilitate the preparation of a speech sequence at a lower processing cost (e.g., Shattuck-

99 Hufnagel, 1979, 1983, 1992; Bohland et al., 2010; Christiansen \& Chater, 2016). At the

100 articulation stage, where those planning units are then "released" to the motor system for

101 articulation, it has also been posited that the motor elements for frequently occurring subsequences

102 become temporally integrated into cohesive sensorimotor programs for producing such

103 subsequences with rapid, highly coordinated movements that have been learned through repeated

104 vocal production practice (e.g., Levelt \& Wheeldon, 1994; Guenther, Ghosh \& Tourville, 2006;

105 Bohland et al., 2010; Segawa, Tourville, Beal \& Guenther, 2015; Guenther, 2016).

106 While researchers generally share the assumption that chunk segmentation and 107 concatenation underlie serial speech planning and production, no consensus has yet been reached

108 regarding the mechanisms that support these processes, or the structure and format of the chunks

109 that these processes operate on (see Bohland et al., 2010; Guenther, 2016, chapter 8, for

110 discussion). Because the type of information represented at each stage of the production planning

111 process likely differs, it is perhaps unsurprising that theories differ in their accounts of the content

112 and size of chunks. Phonological segment error patterns in spontaneous speech, such as the

113 swapping of phonemes in the same phonological position in different planned syllables or words

114 have led to the proposal that frequently produced consonant clusters are, at some level, treated as

115 single units in working memory (e.g., Fromkin, 1971; Shattuck-Hufnagel, 1983, 1992); this is 
116 based largely on the observation that swapping errors often involve entire clusters moving between

117 words (e.g., "dretter swying" for "sweater $\underline{\text { drying"). }}$

118 Results from psycholinguistic experiments using the "word game" paradigm have been

119 interpreted instead as evidence that the fundamental units of phonological working memory and 120 speech sequencing are onset-rhyme units (see, Fowler, Treiman, \& Gross, 1993; cf. Pierrehumbert

$121 \&$ Nair, 1995). For example, in one experiment (Fowler et al., 1993), participants were auditorily

122 presented with trisyllabic pseudowords, and then instructed to repeat, but replace either one (or

123 two) phoneme(s) in the middle-stressed syllable with a fixed sequence. The results were clear in

124 demonstrating that participants responded significantly faster and more accurately when instructed

125 to replace the rhyme of a given syllable, compared to when they were asked to replace the onset 126 and nucleus of a given syllable.

127 Still others have theorized, based on considerations such as coarticulation patterns and 128 syllable frequency effects, that, at the subsequent articulation stage, syllables are the ideal chunk 129 size for speech motor programming units (e.g., Levelt \& Wheeldon, 1994; Cholin et al., 2006; 130 Bohland et al., 2010; Guenther et al., 2006; Guenther, 2016). The fundamental idea here is that, 131 when frequently uttered together, sensorimotor programs for temporally adjacent articulatory 132 gestures fuse together over time to form cohesive action units, which facilitates the production of 133 rapid, highly coordinated movements. On this view, a highly optimized sequence of movements 134 is learned for each frequently produced syllable in the native language.

135 Our recent work on this issue used a motor-sequence learning paradigm in which the 136 generalization of performance gains from practicing novel phoneme sequences was used to infer 137 the nature of speech chunks (Segawa, Masapollo, Tong, Smith, \& Guenther, 2019). In these 138 experiments, native English-speaking participants were trained over several days to produce a set 
of CCVCC non-words involving non-native (i.e., phonotactically-illegal) consonants clusters (e.g.,

140 shkizg, bdepf, dgamj), where $\mathrm{C}$ stands for consonant and $\mathrm{V}$ stands for vowel. The experimental

141 design consisted of two phases: a training phase, during which participants repeatedly produced a

142 set of words, and a subsequent testing phase, during which participants were tested on their ability

143 to produce the previously trained words and a novel set of transfer words. During the training

144 phase of the experiment, we observed that participants progressively executed the target words

145 faster and with increased accuracy, indicative of motor-sequence learning. After learning, we

146 tested for generalization by having participants produce four types of transfer words that

147 systematically varied in their amount of overlap with the trained words: (1) words that were

148 included in their entirety in the training phase (practiced CCVCC), (2) novel words constructed of

149 non-native consonant clusters that were encountered during the training phase (practiced $C C$ ), and

150 (3) novel words containing novel non-native clusters (novel CCVCC) ${ }^{\mathrm{b}}$. The extent to which

151 participants acquired the procedural memories for articulating the trained phoneme sequences

152 during the training phase was evaluated during the testing phase by comparing performance across

153 the trained and novel word types. We found that gains in performance speed from practicing words

154 with non-native consonant clusters largely generalized to the practiced $C C$ words. Practicing the

155 whole word, however, resulted in further performance gains in accuracy over practicing just the

156 clusters.

157 Our interpretation of these findings is as follows. Utterance duration during speech 158 sequence production has long been associated with phonological working memory (PWM) 159 mechanisms (cf. Sternberg, 1978; Klapp, 1995, 2003; Wright, Robin, Rhee, Vaculin, Jacks, 160 Guenther, \& Fox, 2009), which are more taxed when more items need to be stored and retrieved.

\footnotetext{
${ }^{\mathrm{b}}$ Note that this stimulus set was not designed to test whether the rhyme (nucleus-coda) was a representational unit of phonological processing (a la Fowler et al., 1993).
} 
161 Our finding of equal utterance durations for the practiced $C C V C C$ and practiced $C C$ words suggest

162 that these conditions involved the same number of items in PWM. This strongly suggests that the

163 working memory units must not be full syllables - if they were, then the practiced CCVCC

164 condition should have had shorter utterance duration than the practiced $C C$ condition. Instead, the

165 results favor an onset-nucleus-coda (O-N-C) syllabic frame representation (cf. Shattuck-Hufnagel,

166 1992) in which a well-learned consonant cluster constitutes a single item (an onset or coda). Thus,

167 both the practiced $C C V C C$ and practiced $C C$ words consist of three items (or chunks) in PWM,

168 while words containing novel consonant clusters involve five items since consonant cluster units

169 do not exist yet for these words and thus each phoneme from the cluster must be represented as a

170 distinct item.

171 We further hypothesize that error rates are primarily reflective of the articulatory motor

172 programs needed to produce the word rather than PWM mechanisms since working memory

173 demands of producing a single syllable were minimal in the task. Our error rate data indicate that

174 practicing a phoneme sub-string in onset or coda position leads to performance improvements for

175 all syllables containing that sub-string, but that practicing the whole syllable produces even better

176 performance improvements than just practicing the consonant clusters. We account for these

177 results by positing that speech motor programs are learned for any frequently occurring phoneme

178 strings, including not just clusters but also full words/syllables, and the fewer the motor programs

179 that must be sequenced for production, the fewer the errors. Thus, we see the fewest errors for

180 practiced $C C V C C$ words (which involve one syllable-sized motor program), the next fewest for

181 practiced $C C$ words (which require activation of two cluster-sized motor programs and a vowel

182 motor program), and the most errors for novel $C C V C C$ words (which require sequential activation

183 of five phoneme-level motor programs). 
The current research provides a further test of these hypotheses by tackling three issues 185 that were not directly addressed by our prior study (Segawa et al., 2019). First, and perhaps most 186 importantly, we did not provide a definitive test between theoretical accounts that posit that the 187 consonant cluster is a unit of storage in PWM versus a unit of articulation because these two levels 188 of representation were conflated in speakers' productions. In the current study, we attempted to 189 isolate the working memory system from the articulation system by instructing speakers to perform 190 a more demanding version of Segawa et al.'s motor-sequence learning task, in which they were 191 required to maintain more extended sequences of phonemes in memory prior to the onset of 192 production. Since it is harder to remember speech sequences with more items than sequences with 193 fewer items (e.g., Baddeley, Thomson, \& Buchanan, 1975; Acheson \& MacDonald, 2009), we 194 expected to observe different patterns of generalization across conditions for different 195 hypothesized working memory unit types (see detailed predictions below). Disentangling the 196 cognitive structures that operate at the working memory and motor programming levels will not 197 only have important theoretical implications for our understanding of the typical speech production 198 process, but may lead to a method to differentially diagnose PWM versus motor execution 199 impairments in speech sequencing disorders such as apraxia of speech (see also, Buchwald et al., 200 2017).

201 Second, regardless of whether one assumes that the consonant cluster is a PWM unit or an 202 articulatory motor programming unit, the details of such a representation are only partially evident 203 from the results of Segawa et al. (2019) and other studies. Specifically, since a particular cluster 204 in Segawa et al. (2019) always appeared in the same syllable position within a word, it is unclear 205 whether this unit, once learned, generalizes to other syllable positions; for example, does learning 206 of a cluster in the syllable onset position generalize to the same cluster in the coda position? 
207 Findings from other studies (albeit using very different motor tasks) suggest that speech motor 208 learning is often contextually specific (Tremblay, Houle \& Ostry, 2008; Rochet-Capellan, Richer 209 \& Ostry, 2012; cf. Warker \& Dell, 2015). Furthermore, studies focusing on the coordination of 210 articulatory movements for native consonant sequences have shown that the temporal and spatial 211 production of consonants is influenced by syllable position (e.g., Hardcastle, 1985; Byrd, 1996;

212 Loevenbruck, Collins, Beckman, Krishnamurth, \& Ahalt, 1999; Keating, Wright \& Zhang, 1999; 213 Byrd, Lee, Riggs, \& Adams, 2005; Tiede et al., 2007). Moreover, the observation that serial 214 ordering errors in spontaneous speech are greatly biased to occur between phonemes (or clusters) 215 that occupy the same position in different planned syllables or words further bolsters the view that 216 phonological planning representations and/or motor programming units may be positionally 217 constrained (Hindson \& Byrne, 1984; MacKay, 1972; Shattuck-Hufnagel, 1983; Treiman, 1984).

218 A finding that PWM structures or articulatory motor programs for phonemes are sensitive to 219 phonetic context would have important implications for models of speech serial planning and 220 phonetic encoding, as well as for the design of speech motor rehabilitation therapies.

221 Last, but not least, Segawa et al. (2019) focused on motor performance gains without 222 actually measuring the verbal working memory span of participants. Hence, the results only offer 223 speculative support for the role of working memory in task performance. Here we explore whether 224 individual differences in speech motor sequencing show a relation with well-established measures 225 of phonological working memory capacity. Such a finding would be compatible with the view 226 that speakers with a larger working memory capacity can reliably construct and maintain more 227 detailed serial speech plans than those with a smaller working memory capacity (cf. Lee \& 228 Redford, 2015). 
In the current research, we addressed the foregoing issues by using working memory capacity limits to identify the elementary working memory structures involved in serial speech

231 planning and phonetic encoding. As in Segawa et al., we used a cued motor-sequence learning

232 paradigm in which participants repeatedly produced CCVCC words containing non-native onset

233 and coda clusters. Following learning, however, we tested for generalization by having

234 participants repeat four types of pairs $^{c}$ of CCVCC words involving non-native clusters. The use

235 of word pairs, rather than singletons, during the test blocks forced participants to maintain the

236 second word in working memory while the first word was buffered and concurrently articulated.

237 Thus, accuracy and timing measures from the first word, which involved little or no demand on

238 phonological working memory, provided a window into the motor programs used in speech,

239 whereas measures for the second word provided insight into phonological working memory

240 mechanisms. The pairs of words used in the test blocks were carefully constructed such that they

241 systematically differed in their degree of overlap with those practiced in the training phase, and,

242 therefore, also in how much they taxed verbal working memory.

244 training sequences into larger, cohesive chunks in PWM, thereby reducing the processing load and

245 decreasing the sequencing error rate and/or performance speed over time. Indeed, our sequencing

246 studies employing functional magnetic resonance imaging (fMRI), which collected imaging data

247 during the testing phase, after the training phase has taken place outside of the scanner (Segawa et 248 al., 2015; Masapollo, et al., in prep), found greater activation in working memory related regions

249 (inferior frontal sulcus (pIFS), pre-supplementary motor area, anterior insula, and intraparietal

250 sulcus) during the production of novel words compared to trained words. Since sequences with

\footnotetext{
${ }^{\mathrm{c}}$ During pilot testing, we found that prompting participants to produce longer lists of non-native CCVCC words comprised of either three or four items was too challenging a task.
} 
251 more chunks (and hence higher processing loads) are expected to lead to more errors, we expected

252 to observe different error patterns for the second word in the pair across the various experimental

253 conditions for different hypothesized working memory unit types. Specifically, if chunking leads

254 to the formation of a generalized working memory representation of the consonant cluster, then

255 we would expect speakers to exhibit better (and/or faster) performance of novel words that contain

256 the practiced clusters relative to novel syllables with novel clusters. If, however, working memory

257 units code clusters based on their serial position in a word/syllable (i.e., onset versus coda

258 position), then we would expect performance of novel words containing practiced onsets and codas

259 to exhibit better (and/or faster) performance than novel words containing practiced clusters in an

260 unpracticed syllable position. Alternatively, if speakers store and retrieve syllable-sized working

261 memory units, then speakers should exhibit better (and/or faster) performance when producing

262 practiced words but should show no improvements when only the onset/coda clusters are practiced.

\section{2. Materials and Methods}

2642.1 Participants. Sixteen students (6 males, aged 18-25 years, mean age $=20.4$ years $[S D$

$265=2.0]$ ) from Boston University were recruited and paid for participating in two testing sessions.

266 An additional three participants were tested but excluded from analysis due to equipment failure

267 [2] or experimenter error [1]. Participants reported normal (or corrected-to-normal) vision and no

268 history of hearing, speech, language or neurological deficits. All were native, monolingual

269 speakers of standard American English with no previous experience with any of the languages

270 used in the stimulus creation (see following text). We assessed each participant's profile using the

271 Language Experience and Proficiency Questionnaire (LEAP-Q; Marian, Blumenfeld, \&

272 Kaushankskaya, 2007). Participants gave informed consent in accordance with the human-

273 subjects policies of Boston University. 
To characterize participants' verbal working memory capacity, we administered two

275 subtests from the Comprehensive Test of Phonological Processing (CTOPP; Wagner, Torgeson,

$276 \&$ Rashotte, 1999): 1) Memory for digits, which measures the extent to which an individual can

277 repeat a series of numbers (presented auditorily) ranging in length from two to eight digits; 2) Non-

278 word repetition, to measure an individual's ability to repeat non-words (also presented auditorily)

279 that range in length from three to 15 phonemes. To successfully repeat the digits and non-words, 280 an individual must create, store, and maintain a phonological planning representation robust 281 enough to support subsequent articulation.

2.2 Stimuli. The stimuli consisted of four sets of monosyllabic CCVCC non-words (four words/set). As shown in Table 1, all items contained non-native (i.e., phonotactically-illegal)

284 syllable-initial (onset) and syllable-final (coda) consonant clusters. While all of the individual 285 consonants occur in the English phonemic inventory, the particular combination or serial ordering 286 of the segments does not occur in English but does occur in some other natural human language.

287 The non-native clusters were taken from a variety of language and language families including 288 Hebrew, Leti and Taba, Romani, Polish, Lithuanian, Romanian, Georgian, Tepehua, Hungarian, 289 and Pima (see Segawa, 2013; Segawa et al., 2015, for details). None of the participants had prior 290 experience with any of the languages in which these phonotactic sequences are used.

293 standard American English was recorded producing the four sets of words. The model speaker 294 was phonetically trained and had previously practiced producing the sequences until each stimulus 295 could be executed fluently (i.e., without vowel epenthesis or phoneme omissions, swaps, or 296 substitutions). Since we were concerned with learning of non-native phonotactics rather than sub- 
297 phonemic allophonic details, productions were not judged on how natural they sounded in the 298 languages from which they were derived. All recordings took place in a sound-attenuated booth. 299 Speech was recorded directly to a computer using Praat software (Boersma \& Weenink, 2020) via 300 a lapel microphone (Shure, SM93) connected to a pre-amplifier (44.1-kHz sampling rate, 32-bit 301 quantization). The speaker recorded multiple randomized repetitions of each token. From these 302 repetitions, one instance of each token was selected on the basis of acoustic similarity in voice 303 pitch $\left(f_{0}\right)$ to the other stimuli in the set. Using Praat, all recorded tokens were matched for peak 304 intensity and duration (i.e., 480-ms) without changing $f_{0}$.

3052.3 Procedure and design. The experiment consisted of a training phase followed by a 306 transfer test phase. In the training phase, participants were asked to repeat an isolated CCVCC 307 word on each trial. In the test phase, participants were asked to repeat pairs of transfer CCVCC 308 words that overlapped to varying degrees with the those produced in the training phase; in both 309 cases, participants were asked to repeat aloud the word(s) which were presented both auditorily 310 over noise-cancelling headphones and visually using orthography (as shown in Table 1). The 311 training phase consisted of six blocks of trials over two consecutive days (four on day one and two 312 on day two). Each training block contained 10 repetitions of each word, for a total of 40 trials per 313 block. The test phase consisted of two blocks of trials performed on day two after completing the

314 final two training blocks. Each test block contained 10 repetitions of each transfer word pair 315 (shown in Table 1) from four stimulus categories (conditions): (1) practiced CCVCC pairs (i.e., 316 pairs comprised of words that were previously encountered in the training phase), (2) practiced $317 O C$ word pairs (i.e., pairs of novel words that contained non-native consonant clusters that 318 appeared in the same local phonetic context [onset or coda position] in the training words), (3) 319 practiced $C C$ word pairs (i.e., pairs of novel words that contained non-native clusters that appeared 
320 in the training words but in a different syllable position [onset or coda position]), and (4) novel

321 CCVCC word pairs (i.e., pairs of novel words with novel clusters that were not encountered in the

322 training words). This resulted in a total of 80 productions in each test block. Participants produced

323 the target word (or word pairs) in a pseudorandom order. Participants were randomly assigned to

324 one of two experimental groups, which were trained with different CCVCC words. As shown in

325 Table 1, group one was trained with "shkizg", "kshapk", "zvupf," and "bdesch"; group two was

326 trained with "shkupk", "kshezg", "zvisch," and "bdepf." During the subsequent test phase,

327 participants were asked to produce each trained cluster in novel words in both onset position and

328 coda position to assess the contextual specificity of motor-sequence learning.

329 The experiment was programmed in MATLAB (MathWorks Inc., Natick, MA) using the

330 Audio System and Psych Toolboxes. Participants were seated in a chair in front of a laptop

331 (Lenovo ThinkPad X61s) computer screen in a sound-treated laboratory room that was dimly lit.

332 The auditory stimuli were presented over headphones (Sennheiser, HD 280 Pro) at a comfortable

333 listening level and utterances produced by participants were recorded with a lapel microphone

334 (Shure, SM93) connected to the computer via a MOTU Microbook audio interface. Utterances

335 were recorded using MATLAB.

336 Each trial consisted of the following sequence of events. First, the orthographic display of

337 the target word(s) appeared in the center of the screen in tandem with its corresponding auditory

338 prompt. Participants only heard each prompt once. In the training trials, the visual display time

339 for each text stimulus was synchronized with the duration of the associated audio files (i.e., 480-

$340 \mathrm{~ms})$. In the test trials, both text stimuli appeared side-by-side and were displayed for 2,210-ms -

341 this duration was calculated from the onset of the first auditory stimulus to 1,000 -ms after the 
342 offset of the second auditory stimulus (this included an inter-stimulus interval of $250-\mathrm{ms})^{\mathrm{d}}$. Then,

343 after the offset of the visual stimuli, a pure tone was presented for $50 \mathrm{~ms}$. The time between the

344 offset of the visual stimulus and tone onset was randomly jittered between 1,500-ms and 2,000-ms

345 in the training trials and between 2,500-ms and 3,000-ms in the test trials. This tone served as a

346 "Go" signal for the participant to repeat the target word(s) as clearly and accurately as possible.

347 Utterances were recorded for 3,000-ms in the training trials and 5,000-ms in the test trials.

348 Syllables were randomized across trials. We opted to combine the text with the audio presentations

349 because prior studies indicate that listeners tend to perceive non-native consonant clusters as

350 epenthesized disyllabic sequences in an audio-only mode (e.g., Dupoux, Kakehi, Pallier, Hirose,

351 \& Mehler, 1999; Berent, Steriade, Lennertz, \& Vaknin, 2007; Dupoux, Parlato, Frota, Hirose, \&

352 Peperkamp, 2011). Moreover, other research (Davidson, 2010) that directly examined the effects

353 of stimulus input modality (audio only vs. audio and text) on participants' ability to produce non-

354 native consonant clusters found that the presence of text led to an improvement in overall task 355 performance.

356 Participants were instructed to repeat the target word(s) as clearly and accurately as

357 possible as soon as they heard the tone. They were also instructed to attempt to eliminate any

358 vowel-like insertions between consonants within a cluster, a common response when producing

359 novel illegal consonant clusters (cf. Davidson, 2006). Several familiarization trials with

360 experimenter feedback were included at the start of the experiment to confirm that participants

361 understood the task instructions and were able to perform the task. The sequences used during

362 these initial practice trials were not used at any point in the rest of the study.

\footnotetext{
$\mathrm{d}$ Pilot testing revealed that the task during the test phase was too challenging without giving participants additional time (after the offset of the second auditory stimulus) to process the visual text stimuli, suggesting that constraints from reading processes may influence task performance.
} 
Because participants were not provided with online feedback about their performance, they

364 had to learn the novel sequences through a trial-and-error process using a combination of motor 365 exploration and performance evaluation. That is, speakers attempted to produce a given sequence 366 by exploring different methods of moving their articulators, and then evaluated the results of those 367 movements using a combination of auditory and somatosensory perceptual feedback signals. 368 Whether or not the vocal tract maneuvers were ultimately successful in achieving a speech target 369 determined whether it should be repeated.

2.4 Data processing. We used a combination of Praat and custom MATLAB software to 371 perceptually rate and acoustically measure onsets and offsets of syllables by viewing the waveform 372 and spectrogram and listening to the audio files. Each utterance was coded by a trained 373 phonetician, blinded to experimental condition, for seven possible error subtypes: (1) gross 374 disfluency (i.e., productions in which a participant omitted, repeated, or restarted an utterance); (2) 375 unrecognizable from target; (3) phoneme deletion/omission; (4) phoneme insertion (i.e., one or 376 more segments were added); (5) phoneme substitution; (6) incorrect ordering of phonemes; (7) 377 vocoid epenthesis (i.e., truncated vowel-like sounds between consonants within a cluster, 378 evidenced by periodic peaks and a visible second formant in the spectrogram; cf. Wilson, Davidson 379 \& Martin, 2014; Buchwald, Calhoun, Rimikis, Lowe, Wellner, \& Edwards, 2019).

380 As in Segawa et al. (2019), we considered vocoid epenthesis errors to be errors in fluency 381 (in the sense of sounding somewhat different than a native speaker producing the clusters, as if 382 with a strong foreign accent) rather than errors in phoneme sequencing. Previous work examining 383 the production of non-native consonant sequences demonstrates that epenthetic vowels arise from 384 inter-gestural mistiming (Davidson, 2005, 2006; Buchwald, Calhoun, Rimikis, Lowe, Wellner, \& 385 Edwards, 2019); a transitional vowel is produced when the achievement of a consonantal 
constriction does not temporally overlap with the release of the previous tautosyllabic consonantal constriction. Since the primary goal of the current study was to investigate the cognitive processes involved in selecting the proper sequence of motor gestures, rather than the processes involved in

389 articulatory timing, we focused our analyses on sequencing errors (subtypes 1-6). Mean error rates

390 for each participant were calculated as the percentage of trials that contained one or more errors.

In addition to error rates, we also used production timing measures that have been widely

392 used in prior studies (e.g., Sternberg, 1978; Klapp, 1995, 2003; Wright et al., 2009) to probe the

393 nature of working memory sub-units for motor "chunks," namely utterance duration and reaction

394 time (RT). Duration (i.e., time from utterance onset to offset) and RT (i.e., time from the offset of

395 the Go signal to utterance onset) were automatically labeled for each produced word based on

396 sound pressure level thresholds, then hand-checked. For the test trials, we also computed the inter-

397 item-intervals (i.e., time from the offset of the first word production to the onset of the second 398 word production).

399 3. Results

A. Training trials. Our first set of analyses confirmed the presence of motor-sequence 401 learning in the training trials by examining the time course of improvement in three performance 402 measures - sequencing error rates, utterance durations, and RTs - over the two days of speech

403 motor practice. For each word, we computed scores for each performance measure by averaging 404 the values obtained on the first five and last five training trials on day one and the first five training 405 trials on day two. Duration and RT measures were only analyzed for the first or last five utterances 406 coded as properly sequenced productions (see above) on each day. These scores were then pooled 407 and averaged across words within each time point and within each participant. Figure 1 shows the 408 results of these analyses. Separate analyses of variance (ANOVAs) were performed on the mean 
sequencing error rates, durations, and RTs with time as a within-subjects factor (five five trials on

410 day one vs. last five trials on day one vs. first five trials on day two). In these and all subsequent

411 ANOVAs, Greenhouse-Geisser corrections were applied when appropriate and partial eta-squared

412 effect sizes were calculated for all main effects and interactions. Post-hoc pairwise comparisons

413 were reported as significant at the .05 level.

414 Significantly improved performance was found for all three measures, verifying that 415 learning occurred for the training words. Specifically, the ANOVA performed on sequencing error $416 \operatorname{rates}^{\mathrm{e}}$ (shown in Figure 1A) revealed a highly significant main effect of time $[F(2,30)=17.906, p$ $\left.417<.001, \eta_{p}^{2}=0.544\right]$. Post-hoc $t$-tests performed on the pairwise differences indicated that 418 participants produced utterances with fewer errors between the first five $[M=49.6 ; S D=24.5]$ 419 and last five training trials on day one $[M=27.5 ; S D=19.9 ; t(15)=4.745, p<.001, d=0.99]$. 420 These performance gains were retained overnight as mean error rates were not significantly 421 different between the last five training trials on day one and the first five training trials on day two $422 \quad[M=27.5 ; S D=19.7 ; t(15)=.000, p=1.000, d=0]$.

423 The ANOVA performed on utterance durations (shown in Figure 1B) indicated that there 424 was also a significant main effect of time $\left[F(2,30)=3.765, p=.035, \eta^{2} p=0.201\right]$. Post-hoc $t$-tests 425 performed on the pairwise differences indicated that participants got faster at executing their 426 utterances between the first five $[M=.74 ; S D=.10]$ and last five training trials on day one $[M=$ $427.71 ; S D=.09 ; t(15)=2.382, p=.031, d=.31]$, but that the last five training trials on day one and 428 the first five training trials on day two $[M=.70 ; S D=.09]$ were statistically equivalent $[t(15)=$ $429.890, p=.388, d=.11]$. Taken together, these results provide clear evidence that participants

\footnotetext{
e See Supplementary Materials for further details regarding time-course data and analyses of syllable position effects.
} 
430 showed improvements in both performance accuracy and speed over multiple training blocks, and 431 that these gains are retained following an overnight delay without further speech motor practice.

432 For mean RTs (shown in Figure 1C), we again found a significant main effect of time $433\left[F(2,30)=24.747, p<0.001, \eta^{2} p=0.613\right]$. Post-hoc $t$-tests performed on the pairwise differences 434 indicated a reliable learning effect, such that participants got faster at initiating their utterances 435 between the first five $[M=.97 ; S D=.17]$ and last five training trials on day one $[M=.88 ; S D=$ $436.19 ; t(15)=3.469, p=.003, d=.49$ ], and faster still between the last five training trials on day one 437 and the first five training trials on day two $[M=.79 ; S D=.17 ; t(15)=3.338, p=.004, d=.49]$.

438 The latter finding demonstrates that additional off-line improvements in initiation speed occurred 439 over time without practice. Analogous overnight performance enhancements have been reported 440 in a number of motor-sequence learning studies outside of the realm of speech (e.g., finger tapping 441 tasks). The contribution of sleep-mediated memory consolidation to these facilitation effects is 442 supported by a number of studies (e.g., Walker, Brakefield, Morgan, Hobson, \& Strickgold 2002; 443 Walker, Brakefield, Hobson, \& Strickgold, 2003), although this interpretation remains 444 controversial (for discussion, see Brawn, Fenn, Nusbaum, \& Margoliash, 2010; Pan \& Rickard, 445 2015). Nevertheless, given these findings, we then proceeded to examine generalization of these 446 performance gains to the novel, untrained syllables. -- [Insert Figure 1 about here] --

B. Test trials. Our second set of analyses assessed transfer of learning to the novel word 449 pairs in the test phase by examining differences in four dependent measures - sequencing error 450 rates, utterance durations, RTs, and inter-item intervals - between the different experimental 451 conditions (practiced $C C V C C$ vs. practiced $O C$ vs. practiced $C C$ vs. novel $C C V C C$ ). In the 452 analyses performed on sequencing error rates and durations, we also directly compared 
453 performance for the two different stimulus items (first item vs. second item in word pairs) within 454 each condition since the second word is expected to rely most on PWM mechanisms because of 455 its serial position. Table 2 provides the mean frequency of each error subtype in each condition 456 for each stimulus item. As in Segawa et al. (2019), the most common sequencing error was the 457 omission of one or more phonemes in the target syllable.

$458 \quad$ Sequencing error rate. Figure 2 illustrates the mean sequencing error rates pooled and 459 averaged (across subjects) as a function of condition and stimulus item. As the figure shows, 460 participants (unsurprisingly) had some difficulty remembering and producing the pairs of words 461 and generated a high number of sequencing errors. Critically, however, error rates systematically 462 differed across conditions and stimulus items. An ANOVA on these error rates - condition 463 (practiced $C C V C C$ vs. practiced $O C$ vs. practiced $C C$ vs. novel $C C V C C$ ) $\mathrm{X}$ stimulus item (first 464 item vs. second item in syllable pairs $)$ - showed a significant effect of stimulus item $[F(1,15)=$ $\left.46526.241, p<0.001, \eta^{2} p=0.636\right]$ : Participants produced fewer errors when executing the first item $466[M=53.1 ; S D=19.6]$ compared to the second item $[M=72.2 ; S D=18.9]$ in the syllable pairs.

467 This is consistent with other findings in the literature that recall tends to be better for early list 468 items than later list items in verbal working memory tasks (Baddeley, 1986; Burgess \& Hitch, 469 1992). In this case, participants were forced to maintain the second word in working memory 470 during concurrent storage and articulation of the first word, which in turn, may have interfered 471 with storage of the second word. An alternative possibility is that participants may have simply 472 paid a greater amount of attention to the linguistic form of the first word in the planned utterance. 473 The effect of condition was also significant $\left[F(3,45)=27.720, p<0.001, \eta^{2} p=0.649\right]$, as 474 was the predicted interaction $\left[F(3,45)=7.979, p<0.001, \eta^{2} p=0.347\right]$. Simple effects tests on the 475 stimulus item $X$ condition interaction revealed that there was a significant effect of condition on 
476 both the first syllable $\left[F(3,45)=31.844, p<0.001, \eta^{2}{ }_{p}=0.680\right]$ and the second syllable $[F(3,45)$

$\left.477=11.010, p<0.001, \eta^{2}=0.423\right]$. Post-hoc $t$-tests on the pairwise comparisons for the first syllable 478 indicated that the mean error rates for the practiced $C C V C C$ syllables $[M=20.0 ; S D=22.5]$ were 479 significantly lower than the practiced $O C[M=51.8 ; S D=29.9 ; t(15)=-5.193, p<.001, d=1.2]$, 480 practiced $C C[M=59.3 ; S D=28.6 ; t(15)=-6.498, p<.001, d=1.5]$, and novel $C C V C C$ syllables $481[M=81.2 ; S D=16.6 ; t(15)=-13.453, p<.001, d=3.0]$. In addition, the mean error rates for the 482 practiced $O C$ syllables were significantly lower than the novel CCVCC syllables $[t(15)=-4.825$, $483 p<.001, d=1.2$ ], and the mean error rates for the practiced $C C$ syllables were lower than the 484 novel CCVCC syllables $[t(15)=-3.732, p=.002, d=0.93]$. Although, the practiced $O C$ and 485 practiced $C C$ error rates were not significantly different from each other $[t(15)=-.864, p=.401$, $486 d=.25]$, there was a small benefit to having practiced producing the clusters in the same syllable 487 position. Overall, the error rate results for the first syllable mirror our prior findings using only a 488 single test syllable (Segawa et al., 2019): syllables practiced in their entirety show the lowest error 489 rates, while practicing only a subset of the syllable (in this case the consonant cluster in either the 490 same or different syllable position) resulted in somewhat lower error rates than fully novel 491 syllables but higher error rates than fully practiced syllables.

493 the practiced $C C V C C$ syllables $[M=49.3 ; S D=36.6]$ were significantly lower than the practiced $494 O C[M=73.1 ; S D=26.5 ; t(15)=-2.911, p=.011, d=.74]$, practiced $C C[M=90.6 ; S D=15.2 ;$ $495 t(15)=-4.522, p<.001, d=1.47]$, and novel $C C V C C$ syllables $[M=75.6 ; S D=20.3 ; t(15)=-$ $4963.992, p=.001, d=.88$ ]. However, practicing a subset of the syllable did not improve error rates 497 over fully novel syllables: the practiced $O C$ and novel $C C V C C$ error rates were not significantly 498 different from each other $[t(15)=-.389, p=.703, d=.10]$, and the error rate for the practiced $C C$ 
499 syllables was actually higher than that for the novel CCVCC syllables $[t(15)=2.818, p=.013, d$

$500=.83]$. In addition, the mean error rates for the practiced $O C$ syllables were significantly lower

501 than the practiced $C C$ syllables $[t(15)=-2.369, p=.032, d=.81]$. Collectively, these results

502 suggest that consonant clusters are positionally encoded in phonological working memory

503 structures but not motor programming units.

504 Duration of individual motor speech elements. As already discussed, production timing

505 measures during sequence production have been associated with working memory processes (e.g.,

506 Sternberg, 1978; Klapp, 1995; Wright et al., 2009), which are more taxed when more sub-units (or

507 chunks) need to be concatenated and stored. We therefore reasoned that temporal aspects of

508 sequence generation (i.e., utterance duration and initiation speed) should increase with the number

509 of subunits (in our case, the number of sub-units in each word/syllable) in PWM.

510 As can be seen in Figure 2, participants often produced a sequencing error on one of the

511 stimulus items within a given syllable pair, especially for the practiced $C C$ and novel CCVCC

512 conditions, which severely limited the number of properly sequenced productions that could

513 contribute to the temporal measurements. To increase our statistical power, we opted to examine

514 generalization of the production timing measures based on the mean durations, RTs, and inter-

515 item-intervals obtained for each word pair on the first five test trials, regardless of whether both

516 items were properly sequenced or not. This provided a gross measure of how comfortable

517 participants were with producing these non-native sequences following training. Durations were

518 pooled and averaged within each condition and stimulus item and within each subject. RTs and

519 inter-item intervals were also all pooled and averaged within each condition and within each

520 subject. 
Figure 3 shows the mean utterance durations (across subjects) as a function of condition.

522 The ANOVA performed on mean durations revealed significant main effects of stimulus item $523\left[F(1,45)=18.069, p=.001, \eta_{p}^{2}=0.546\right]$, such that the first syllable was executed faster $[M=.65$ $524 S D=.10]$ than the second syllable $[M=.72 ; S D=.12]$. There was also a main effect of condition $525\left[F(3,45)=3.433, p=.025, \eta_{p}^{2}=0.186\right]$ and a significant interaction effect $[F(3,45)=7.754, p<$ $\left.526 \quad .001, \eta^{2}=0.341\right]$

Simple effects tests on the stimulus item $\mathrm{X}$ condition interaction revealed that there was a 528 significant effect of condition on the performance speed of the second syllable $[F(3,45)=9.681$, $\left.529 p<0.001, \eta_{p}^{2}=0.392\right]$, but not the first syllable $\left[F(3,45)=1.216, p=0.315, \eta_{p}^{2}=0.075\right]$. The 530 lack of a significant duration difference between conditions for the first syllable is somewhat 531 surprising given that our prior study using single test syllables (Segawa et al., 2019) found that 532 practicing either the full syllable or the onset or coda cluster resulted in lower durations than fully 533 novel syllables. Overall, mean utterance duration for the first syllable in the current study was 534 longer than for the isolated syllable in the prior study $[t(25)=-2.169, p=.040, d=.89]$. The duration pattern for the second syllable in the current study mirrored those for the 536 single syllable in our prior study, namely that practicing onset and coda clusters led to duration 537 reductions compared to fully novel syllables that were statistically indistinguishable from the 538 duration reductions for fully practiced syllables. Specifically, follow-up $t$-tests on the pairwise 539 comparisons for the second syllable indicated that the mean durations for the practiced CCVCC 540 syllables $[M=.67 ; S D=.10]$ were significantly faster than the practiced $C C[M=.75 ; S D=.11$; $541 t(15)=-5.417, p<.001, d=.76]$ and novel CCVCC syllables $[M=.77 ; S D=.14 ; t(15)=-3.895$, $542 p<.001, d=.82]$. However, the differences between the mean durations for the practiced CCVCC 543 syllables and practiced $O C$ syllables $[M=.70 ; S D=.11]$ did not reach significance $[t(15)=-1.852$, 
$544 p=.084, d=.28]$. In addition, the mean durations for the practiced $O C$ syllables were significantly

545 faster than both the practiced $C C$ syllables $[t(15)=-3.281, p=.005, d=.39]$ and the novel CCVCC

546 syllables $[t(15)=-2.591, p=.020, d=.55]$. The practiced $C C$ and novel $C C V C C$ durations were

547 not significantly different from each other $[t(15)=-.740, p=.471, d=.15]$.

Reaction time and inter-item-interval. Figure 4 shows the mean RTs and inter-trialintervals (across subjects) as a function of condition. An ANOVA analysis of RT scores with

551 experimental condition (practiced $C C V C C$ vs. practiced $O C$ vs. practiced $C C$ vs. novel $C C V C C$ )

552 as a within-subjects factor was performed on utterances produced during the first five test trials of

553 each condition. There was no main effect of condition $\left[F(3,45)=1.920, p=.140, \eta^{2} p=0.113\right]$,

554 indicating that the reaction time for producing the first syllable did not significantly depend on 555 condition. In contrast, an ANOVA analysis of inter-trial intervals revealed a highly significant 556 main effect of condition $\left[F(3,45)=7.383, p<.001, \eta^{2}{ }_{p}=0.330\right]$. Post-hoc $t$-tests performed on 557 these pairwise differences indicated that participants initiated the second stimulus item faster for 558 the practiced $C C V C C$ syllables $[M=.32 ; S D=.16]$ than the practiced $C C[M=.40 ; S D=.13$; $559[t(15)=-3.441, p=.004, d=.54]$ and novel $C C V C C$ syllables $[M=.43 ; S D=.14 ; t(15)=-3.540$ $560 p=.003, d=.74]$, but not for the practiced $O C$ syllables $[M=.35 ; S D=.17 ; t(15)=-1.601, p=$ $561.130, d=.18]$. Inter-item-intervals for the practiced $O C$ syllables were also significantly faster 562 than the novel CCVCC syllables $[t(15)=-2.485, p=.025, d=.51]$ but not significantly faster than 563 the practiced $C C$ syllables $[t(15)=-1.847, p=.085, d=.33]$. In sum, the pattern of the delay prior 564 to onset of the second syllable (the inter-trial interval) across conditions mirrored the pattern for 565 second syllable duration: practicing onset and coda clusters led to inter-trial interval reductions 
compared to fully novel syllables that were statistically indistinguishable from the inter-trial interval reductions for fully practiced syllables.

Duration of entire sequence of motor speech elements. In much of the literature on working memory and motor-sequence production (e.g., Sternberg, 1978; Klapp, 1995; Wright et al., 2009),

571 the focus has been on RT before the first element, as well as overall duration of the entire sequence 572 of motor elements (rather than the duration of individual elements, as reported above). The data 573 and arguments from these studies (albeit using different paradigms and tasks) have given rise to 574 two main claims: (1) RT for the first item does not depend on the number of working memory sub575 units (or "chunks") in each item, since the motor program for the first item can be retrieved and 576 unpacked prior to the onset of the Go signal ${ }^{\mathrm{f}}$, and (2) overall sequence duration should increase 577 with the number of sub-units in working memory. The first claim, that RT should be the same 578 across all experimental conditions, was confirmed in both the present experiment (Figure 3, left 579 panel) as well as in our prior study (Segawa, et al., 2019).

As for the second claim, we know this to be the case from Segawa, et al. (2019) since 581 overall duration was the same as element duration in that experiment (i.e., participants produced 582 singletons rather than word pairs during test). Recall, in that study, that the duration for practiced $583 C C V C C$ and practiced $O C$ words were statistically equivalent to one another, and shorter than that 584 of the novel CCVCC words. This finding is compatible with the view that well-learned consonant 585 clusters constitutes a single item (an onset or coda) in PWM; both the practiced CCVCC and practiced $O C$ words consist of three working memory elements, while words containing novel

\footnotetext{
${ }^{\mathrm{f}} \mathrm{RT}$ in a choice reaction time paradigm, on the other hand, would depend on the number of sub-units in the syllable since the sub-units cannot be unpacked until the Go signal indicates which syllable to produce (Klapp, 1995; Klapp, 2003).
} 
consonant clusters involve five items since consonant cluster units do not exist yet for these words and thus each phoneme from the cluster must be represented as a distinct item.

To examine further whether this is the case for the word pairs in the present experiment,

590 we conducted an additional analysis in which we computed the mean overall duration of word

591 pairs during test trials as a function of experimental condition (practiced CCVCC vs. practiced

$592 O C$ vs. practiced $C C$ vs. novel $C(V C C)$. Overall duration was calculated by summing the duration

593 of the first word, the inter-item-interval, and the second word duration. Figure 5 [left panel] shows

594 the results of this calculation based on participants' first five production attempts, regardless of 595 whether the utterance was properly sequenced. An ANOVA conducted on these duration measures 596 with experimental condition as a within-subjects factor revealed a main effect of condition $597\left[F(3,45)=8.404, p<.001, \eta^{2} p=0.359\right]$. Post-hoc $t$-tests revealed a significant difference between 598 the mean duration of practiced CCVCC words pairs $[M=.1 .62 ; S D=.26]$ and practiced $\mathrm{CC}[M=$ $5991.81 ; S D=.28 ; t(15)=-4.352, p=.001, d=-.58]$ and novel CCVCC word pairs $[M=1.82 ; S D=$ $600.28 ; t(15)=-4.044, p=.001, d=-.61]$. The practiced $O C[M=1.71 ; S D=.32]$ and practiced $C C$ 601 durations were also significantly different from each other $[t(15)=-2.285, p=.037, d=-.26]$. All 602 other post-hoc comparisons were not significant $(p>.05)$. Additionally, Figure 5 [right panel] 603 shows the results of this calculation based only on the first five production attempts that were 604 perceptually judged to be properly sequenced, although there was insufficient data to perform 605 formal statistical tests. Nevertheless, the results of the aforementioned analyses further bolster the 606 view that practiced $C C V C C$ and practiced $O C$ words contain three chunks in PWM, corresponding 607 roughly to onset-nucleus-coda, whereas novel CCVCC words contains five chunks in PWM, 608 corresponding to individual phonemes. 
611 the relationship between standardized measures of phonological working memory capacity and 612 overall task performance in the test phase. Correlational analyses among each of the CTOPP digit 613 recall and non-word repetition scores and the mean number of sequencing errors for each stimulus

614 item (first word vs. second word) were performed. No correlations emerged among the digit recall 615 scores and the mean number of sequencing errors produced on either the first item $(r=-.34, p=$ $616.19, n=16)$ or second item in the word pairs $(r=-.36, p=.16, n=16)$.

617 The correlation among the non-word repetition scores and the mean number of sequencing 618 errors produced was significant for the second word $(r=-.60, p=.01, n=16)$ but not for the first 619 word $(r=-.34, p=.19, n=16)$. A Fisher's $r$-to- $z$ transformation was then performed to test for a 620 potential difference in the size of these two correlations. Using either a one-tailed or two-tailed 621 test of significance, these correlations were not found to significantly differ $(z=-0.86, p>.05)$, 622 however, contra the hypothesis that production of the second syllable is more dependent on PWM 623 mechanisms. Furthermore, it is important to note that because participants produced more 624 sequencing errors on the second word than the first word (Figure 2), there is also more variation 625 and hence more statistical power to observe a correlation. Although the foregoing results do not strongly support the notion that the production of the

627 second word relies more heavily on phonological working memory than the first word, we should 628 be careful not to draw firm conclusions from these analyses. It is possible that there simply were 629 not a sufficient number of participants to have the power to detect a difference in size between the 630 correlations. Another possibility is that we may need to use a more thorough test of verbal working 631 memory (and potentially more stimuli) to capture meaningful differences.

\section{4. Discussion}


In the current study, we investigated the degree to which components of newly learned

634 words containing non-native consonant clusters (practiced one at a time) generalized to transfer

635 word pairs. The primary difference between production of the first and second words in the test

636 phase of our protocol is that the second syllable must be held in PWM while the first syllable is

637 being produced. Therefore, performance differences across conditions for the second syllable

638 compared to the first presumably reflect properties of PWM mechanisms. Within this view, the

639 present error rate results strongly imply that PWM differentiates between a consonant cluster in

640 one syllabic position (onset or coda) and the same cluster in a different syllabic position. In fact,

641 when participants were asked to produce a practiced cluster in a different syllable position, their

642 performance deteriorated; this interference effect can be seen by the higher error rate for the

643 practiced $C C$ condition compared to the practiced $O C$ and novel $C C V C C$ conditions for the second

644 word (Figure 2). Furthermore, improvements in the duration of the second word were seen only

645 for words in which novel clusters appear in the same location as they appeared in the training

646 phase. This is evidenced by the finding that second syllable durations in the practiced CCVCC

647 and practiced $O C$ conditions are shorter than for the practiced $C C$ condition, with the practiced

$648 C C$ condition showing no improvement in duration over fully novel (novel CCVCC) syllables

649 (Figure 3). The same pattern was seen in the analyses of the inter-trial intervals (basically

650 equivalent to second syllable reaction time in our protocol; see Figure 4) and overall duration of

651 the word pairs (see Figure 5), again highlighting the importance of syllabic position constraints on

652 PWM. Such findings suggest that, during the early stages of speech motor-sequence learning,

653 adjacent tautosyllabic consonants bind together into unified working memory units that are

654 phonologically differentiated (onset vs. coda). 
In contrast to the second syllable results, error rates for the first syllable exhibit a pattern indicating that improvements from practicing a consonant cluster in one syllable position

657 generalize to novel syllables in which that cluster appears in a different syllable position 658 (evidenced by equal error rates for the practiced $O C$ and practiced $C C$ conditions in Figure 2), 659 with both of these conditions showing less improvement in accuracy than practicing the entire 660 syllable (practiced CCVCC in Figure 2). These findings support the view that, unlike working 661 memory structures, the motor programming units for speech production are not affected by the 662 syllable position in which a newly learned phoneme subsequence (in this case a non-native 663 consonant cluster) is learned; this "motor knowledge" can be applied any time the subsequence 664 appears in future utterances, regardless of syllable position. Thus, PWM appears to utilize an 665 onset-nucleus-coda syllabic frame structure, whereas the motor system appears to use a syllable666 frame-independent representation of common phoneme sub-sequences.

667 Figure 6 provides a possible account of these findings, based on the gradient order DIVA 668 (GODIVA) model of speech sequencing advanced by Guenther and his colleagues (Bohland et al., 669 2010; Guenther, 2016). According to this model, the to-be-articulated segments in a planned 670 utterance are temporarily stored in a phonological working memory repository (or buffer). During 671 the production of native-language speech, syllables are represented by up to three chunks in this 672 repo: an onset consisting of one or more initial consonants, a nucleus (vowel), and a coda 673 consisting of one or more final consonants (O-N-C representation). We hypothesize that this 674 representation is used for any syllable that utilizes native consonant clusters or highly-practiced 675 non-native clusters, as schematized in the practiced $C C V C C$ and practiced $O C$ conditions of 676 Figure 6 for the phonotactically illegal syllable BDEPK. When producing a syllable containing 677 novel non-native clusters, the system is forced to represent each phoneme individually in PWM 
678 (novel CCVCC condition in Figure 6). Thus, a fully novel CCVCC syllable requires five chunks

679 in PWM compared to three for novel CCVCC syllables that utilize learned clusters and three for

680 fully learned CCVCC syllables. Utterance duration in GODIVA depends on syllable complexity,

681 specifically the number of chunks (or motor elements) for each syllable that must be retrieved and

682 unpacked from PWM prior to production of the syllable, a relationship supported by numerous

683 prior motor sequencing studies (e.g., Sternberg, 1978; Klapp, 1995, 2003; Wright et al., 2009).

684 According to this account, utterance duration should be longest for the novel CCVCC condition,

685 with the practiced $O C$ and practiced CCVCC conditions having the same duration; indeed, this is

686 the pattern we found for utterance duration in both the current study and Segawa et al. (2019).

687 Furthermore, because this account differentiates phonological and motoric learning processes, it

688 may help explain why some individuals with apraxia of speech (AOS), namely those with

689 primarily motoric deficits, improve their productions of consonant clusters with practice, while

690 individuals with AOS who display primarily phonological deficits do not (Buchwald et al., 2017).

-- [Insert Figure 6 about here] --

However, even if this account of serial speech sequencing turns out to be correct, we are

693 still left with the deeper question as to why PWM structures, but not the motor programming units,

694 appear to be contextually specified. One possibility is that higher-level working memory processes

695 are shaped and constrained by linguistic experience to a greater degree than lower-level

696 articulatory processes. Phonological units differ across languages and the brain becomes fine-

697 tuned to the units of the language of exposure early in development (Kuhl, Ramirez, Bosseler, Lin,

698 \& Imada, 2014), and although some aspects of articulatory behavior are clearly affected by specific

699 linguistic experience (e.g, Byrd et al., 2005; Beddor, Harnsberger, \& Lindemann, 2002; Tiede et

700 al., 2007), humans (and non-human animals) can (and must) learn to execute motor programs for 
701 a wide range of sequential motor behaviors (e.g., typing keyboard sequences, playing a sport or 702 musical instrument) that are not constrained by linguistic structure or rules.

703 In the current study, both duration and error rates were significantly lower for words 704 practiced in their entirety during the training sessions compared to fully novel syllables. However, 705 for novel syllables consisting of non-native consonant clusters that were practiced but in different 706 syllables, error rates were significantly higher than for fully practiced syllables, while duration for 707 these stimuli was just as low as for fully practiced sequences. The GODIVA model accounts for 708 this difference by positing that duration measures are indicative of the number of chunks for each 709 sequence in PWM, whereas articulatory error rates are indicative of the number of motor programs 710 needed to produce the sequence. However, these findings also raise the possibility that there may 711 be a dissociation between learning how to execute articulatory timing information (as measured 712 by duration) and the proper sequence of articulatory motor gestures (as measured by error rates).

713 That is, different aspects of sequence production may be learned on different time-scales ${ }^{\mathrm{g}}$ and 714 mediated by different cognitive processes and neural substrates. Interestingly, the GODIVA 715 model does propose that prefrontal medial areas (left pre-supplementary motor area, left 716 supplementary motor area) are recruited in coordinating the speech articulators in time to achieve 717 motor goals in sequence, while lateral areas (left inferior frontal sulcus, left ventral premotor 718 cortex) are thought to be recruited to generate speech motor programs for articulation. Further 719 studies using an integrated combination of articulatory kinematic and functional brain-imaging 720 methods are needed to provide a direct test of this claim, however, and isolate the neural substrates 721 underlying articulatory errors versus utterance duration.

\footnotetext{
${ }^{\mathrm{g}}$ See Supplementary Materials for additional information on the time-course of speech motor-sequence learning.
} 
One unexplained aspect of our findings is that of why we failed to observe any reliable

723 timing differences across conditions for the first syllable (shown in Figure 3), unlike in Segawa et

724 al. (2019). Our task was identical to that one used by Segawa and colleagues except that

725 participants also had to buffer a subsequent word during concurrent articulation of the first word.

726 Thus, somehow the timing differences documented by Segawa et al. washed out when there was

727 a subsequent word stored in PWM. From our second syllable results, we reasoned that gains in

728 performance speed seem to have to do with PWM processes. Thus, perhaps the "first" syllable in

729 the Segawa et al. study was held in working memory longer but was processed more rapidly in the 730 current study in order to minimize interference from the second syllable.

731 As well, the finding that, practicing a consonant cluster in one syllable position generalizes

732 to novel syllables in which that cluster appears in a different syllable position (Figure 2 [left

733 panel]), runs contra to previous reports that speech motor learning is contextually specific

734 (Tremblay et al., 2008; Rochet-Capellan et al., 2012), and is somewhat surprising given that the 735 temporal and spatial production of (native-language) consonant clusters (and their corresponding 736 sensory outcomes) is known to be affected by syllable position (e.g., Hardcastle, 1985; Byrd, 1996;

737 Loevenbruck et al., 1999; Keating et al., 1999; Byrd et al., 2005; Tiede et al., 2007). We might,

738 therefore, speculate that during speech motor practice, participants learn higher-level patterns of 739 coupling or coordination among articulators that can be flexibly assembled across different 740 phonetic contexts.

741 Although cluster production was found to generalize to novel syllables containing those 742 clusters, the results also showed that participants produced roughly twice as many errors in the 743 practiced $O C$ and practiced $C C$ conditions compared to the practiced $C C V C C$ condition (see 744 Figure 2). There are at least two possible explanations for this later finding. One possibility is 
745 that, during the initial stages of speech motor-sequence learning, speakers bind together the 746 individual vocal tract movements associated with adjacent consonants into a unified action, or 747 motor "chunk," but that larger syllable-sized chunks are eventually formed in addition to 748 consonant cluster-sized units. An alternative possibility, however, is that the relevant sequencing 749 sub-units or chunks are actually onset-vowel or vowel-coda (rhyme) units. Indeed, there is a body 750 of evidence supporting the later possibility.

Research on the interface between linguistic representation and speech production has

752 demonstrated that the relative timing and stability of articulatory gestures (for native-language 753 sequences and structures) changes systematically as a function of numerous contextual factors.

754 One of these is syllable position (e.g., Browman \& Goldstein, 1988; Byrd, 1996; Byrd et al., 2005;

755 Tiede et al., 2007). Early research, using X-ray microbeam data, found that the temporal 756 coordination between syllable-initial (onset) consonants and the syllable's vowel differs from that 757 of syllable-final (coda) consonants and the syllable's vowel (Browman \& Goldstein, 1988). In 758 particular, it was found that the timing of the onset as a whole with respect to the vowel remained 759 relatively stable, regardless of the number of consonants and the composition of a given onset 760 cluster (i.e., "C-center" effects). In contrast, this cluster-vowel timing pattern did not emerge with 761 codas. Subsequent studies, using both electropalatography (EPG) and electromagnetic 762 articulography (EMA), obtained similar patterns with several onset clusters and languages and also 763 showed that onset clusters tend to be implemented with less variable inter-gestural timing than 764 coda clusters (e.g., Byrd, 1996; Byrd et al., 2005; Pastatter \& Pouplier, 2017). On the basis of 765 these findings, some theoretical accounts have proposed that the motor programs for onsets and 766 vowels are fused together as cohesive action units, or motor "chunks," at least at some level of the 767 speech production process (for discussion, see Goldstein, Byrd, \& Saltzman, 2006; Pastatter \& 
768 Pouplier, 2017). Such findings are relevant to the current research in that is unknown whether one 769 could transfer learning of non-native cluster coordination from onsets to codas and vice versa. 770 Unfortunately, however, we are unable to directly address this issue without detailed, kinematic 771 measures of the participants' articulatory movements, although we have begun to investigate 772 gestural coordination for non-native productions in ongoing experiments using electromagnetic 773 articulography (Cheng, Masapollo, Hagedorn, \& Buchwald, in progress; Masapollo, Kearney, \& 774 Guenther, in progress). Additionally, a strong test of the so-called "C-center" hypothesis in the 775 present context would require additional stimulus conditions, including practiced $C C V$ and 776 practiced VCC words. Future experiments will be designed with this issue in mind.

778 changes in motor performance using perception-based segmental transcription and simple acoustic 779 measures of utterance duration. However, this approach requires transcribers to make categorical 780 decisions regarding the segments that speakers produce, and thus does not permit quantitative 781 analysis of the presence or magnitude of various articulatory gestures. In other experimental 782 studies designed to examine the nature of speech errors in both normal and disordered speakers 783 (albeit using different speaking tasks and stimulus materials), researchers have provided 784 compelling evidence that errors are often partial or gradient (see, e.g., Mowrey \& MacKay, 1990; 785 Goldrick \& Blumstein, 2006; Goldstein, Pouplier, Chen, Saltzman, \& Byrd, 2006; Hagedorn, 786 Proctor, Goldstein, Wilson, Miller, Gorno-Tempini, \& Narayanan, 2017). Kinematic data from 787 tongue and lip movements during sequencing errors elicited in our task could help provide further 788 insights into whether such errors reflect limitations in PWM or incomplete control of gestural 789 coordination. If errors derive from immature motor implementation rather than a limited working 790 memory store, then we predict that speakers will generate truncated vocal tract constrictions 
791 (which may be acoustically masked to transcribers). Else we predict that errors arise at a higher

792 organizational level of speech planning whereby sequences of phonological units are assembled

793 and temporarily buffered in the phonological store.

794 The transcription methods used in the current study also provide a limited window on 795 speech motor learning itself since they provide an indirect measure of articulator movement 796 characteristics among cluster elements (such as inter-gestural timing) and how they change as a 797 function of speech motor practice. Moreover, other research on speech production indicates that 798 the intended goals (or "targets") of speech production are somatosensory, as well as acoustic, in 799 nature (e.g., Tremblay, Shiller, Ostry, 2003; Golfinopoulos, Tourville, Bohland, Ghosh \& 800 Guenther, 2011), and that co-articulatory overlap can weaken or even obliterate acoustic cues for 801 consonants (especially plosives) produced in clusters (Stevens \& Keyser, 2010; Perkell, 2012). A 802 complete understanding of speech motor-sequence learning, therefore, will require data on both 803 acoustics and articulation. Ongoing work in our laboratory using electromagnetic articulography 804 is aimed at characterizing how the articulatory activity that occurs within the inner reaches of the 805 vocal tract dynamically changes during motor-sequence learning.

806 In conclusion, the present findings add to a vast and illuminating body of literature 807 examining how speakers represent, plan and execute sequences of speech movements (as recently 808 reviewed in Guenther, 2016). In moving toward a theory of language and speech production that 809 fully explicates the organization and execution of speech sequences, future work should consider 810 how working memory structures and sensorimotor programs for speech might differ across human 811 languages, especially those that do not have tautosyllabic consonant clusters and/or a phonetically812 transparent orthographic writing system (e.g., Japanese or Mandarin). Is it possible that the mere 813 presence of text stimuli in our procedure and the one used by Segawa et al. (2019) may have biased 
814 speakers to "visually chunk" or segment non-native phoneme sequences in a particular way. In 815 additional to potential reading processing effects, it is also not yet known whether and how these 816 units might vary in pre-literate children learning to talk, or in speakers with disorders of speech 817 sequencing (e.g., stuttering, apraxia of speech). Future studies in our laboratory will focus on 818 addressing these issues. 


\section{Acknowledgements}

820 The research reported here was supported by a grant from the National Institutes of Health (R01

821 DC007683; F.H. Guenther, PI). The content is solely the responsibility of the authors and does

822 not necessarily represent the official views of the National Institutes of Health. We are grateful to

823 Barbara Holland, Farwa Faheem, Tess Fairchild, Kaylee Tran, and Riccardo Falsini for assistance

824 with subject recruitment, data collection and analysis. This work benefited from helpful

825 discussions with, or comments from, Jason Bohland, Jason Tourville, Stefanie Shattuck-Hufnagel,

826 Gary Dell, and audience members at the 2019 Boston Speech Motor Control Symposium and at

827 the $178^{\text {th }}$ Meeting of the Acoustical Society of America. 


\section{References}

829 Acheson, D.J., \& MacDonald, M.C. (2009). Verbal working memory and language production: common approaches to the serial ordering of verbal information. Psychological Bulletin,

832 Baddeley, A. D., Thomson, N., \& Buchanan, M. (1975). Word length and the structure of shortterm memory. Journal of Verbal Learning \& Verbal Behavior, 14, 575-589.

834 Baddeley, A. D. (1986). Working memory. New York: Clarendon Press/ Oxford University Press.

835 Beddor, P.S., Harnsberger, J.D., Lindemann, S. (2002). Language-specific patterns of vowel-to-

836 vowel coarticulation: acoustic structures and their perceptual correlates. Journal of $837 \quad$ Phonetics, 30(4), 591-627.

838 Berent, I., Steriade, D., Lennertz, T., \& Vaknin, V. (2007). What we know about what we have never heard: Evidence from perceptual illusions. Cognition, 104, 591-630.

840 Bohland, J. \& Guenther, F.H. (2006). An fMRI investigation of syllable sequence production. NeuroImage, 32(2), 821-841.

Bohland, J.W., Bullock, D., \& Guenther, F.H. (2010). Neural representations and mechanisms for the performance of simple speech sequences. Journal of Cognitive Neuroscience, 22(7), pp. $1504-1529$.

Boersma, P. \& Weenink, D. (2020). Praat: doing phonetics by computer [Computer program]. Version 6.1.09, retrieved 26 January 2020 from http://www.praat.org/

Botvinick, M. M., \& Plaut, D. C. (2006). Short-term memory for serial order: A recurrent neural network model. Psychological Review, 113, 201-233.

Brawn, T.P., Fenn, K.M., Nusbaum, H.C., \& Margoliash, D. (2010). Consolidating the effects of 
waking and sleep on motor-sequence learning. Journal of Neuroscience, 30(42), 1397713982.

852 Browman, C. \& Goldstein, L. (1988). Some Notes on Syllable Structure in Articulatory Phonology. Phonetica, 45, 140-155.

854 Buchwald, A., \& Miozzo, M. (2012). Phonological and motor errors in individuals with acquired impairment. Journal of Speech, Language and Hearing Research, 55(5), 1573-1586.

Buchwald, A., Gagnon, B., \& Miozzo, M. (2017). Identification and remediation of phonological and motor errors in acquired sound production impairment. Journal of Speech, Language,

Buchwald, A., Calhoun, H., Rimikis, S., Lowe, M.S., Wellner, R., \& Edwards, D.J. (2019). Using tDCS to facilitate motor learning in speech production: The role of timing. Cortex, 111, 274-285.

Burgess, N., \& Hitch, G. J. (1992). Toward a network model of the articulatory loop. Journal of Memory and Language, 31, 429-460.

Byrd, D. (1996). Influences on articulatory timing in consonant sequences. Journal of Phonetics,

Byrd, D., Lee, S., Riggs, D., Adams, J. (2005). Interacting effects of syllable and phrase position on consonant articulation. Journal of the Acoustical Society of America, 118(6), 38603873.

Cai, S., Ghosh, S.S., Guenther, F.H., and Perkell, J.S. (2011). Focal manipulations of formant trajectories reveal a role of auditory feedback in the online control of both within-syllable

872 Christiansen, M.H., \& Chater, N. (2016). The now-or-never bottleneck: a fundamental constraint 
on language. Behavioral and Brain Sciences, 1-72. doi:10.1017/S0140525X1500031X, e62.

875 Cholin, J., Levelt, W.J.M., \& Schiller, N.O. (2006). Effects of syllable frequency in speech $876 \quad$ production. Cognition, 99, 205-235.

877 Davidson, L. (2006). Phonology, phonetics, or frequency: Influences on the production of non878 native sequences. Journal of Phonetics, 34, 104-137.

879 Davidson, L. (2010). Phonetic bases of similarities in cross-language production: Evidence from English and Catalan. Journal of Phonetics, 38, 2, 272-288.

881 Dell, G.S. (1986). A spreading-activation theory of retrieval in sentence production. Psychological Review, 93(3), 283-321.

Dell, G.S., Juliano, C., \& Govindjee, A. (1993). Structure and content in language production: A theory of frame constraints in phonological speech errors. Cognitive Science, 17, 149-195.

Dupoux, E., Kakehi, K., Pallier, Y., Hirose, C., \& Mehler, J. (1999). Epenthetic vowels in Japanese: a perceptual illusion? Journal of Experimental Psychology: Human Perception and Performance, 25(6), 1568-1578.

890 Fenn, K.M., Nusbaum, H.C., \& Margoliash, D. (2003). Consolidation during sleep of perceptual leaning of spoken language. Nature, 425, 614-616.

892 Fowler, C.A. (1987). Consonant-vowel cohesiveness in speech production as revealed by initial and final consonant exchanges. Speech Communication, 6, 231-244.

894 Fowler, C.A., Treiman, R., \& Gross, J. (1993). The structure of English syllables and polysyllables. Journal of Memory and Language, 32, 115-140. 
Fromkin, V.A. (1971). The non-anomalous nature of anomalous utterances. Language, 47, 1, 27 52.

898 Goldstein, L., Pouplier, M., Chen, L., Saltzman, E., \& Byrd, D. (2007). Dynamic action units slip in speech production errors. Cognition, 103(3), 386-412.

Goldrick, M., \& Blumstein, S. (2006). Cascading activation from phonological planning to

Golfinopoulos E., Tourville J.A., Bohland J.W., Ghosh S.S., Guenther F.H. (2011). fMRI of unexpected somatosensory feedback perturbation during speech. NeuroImage, 55, 13241338.

Guenther, F.H., Ghosh, S.S., and Tourville, J.A. (2006). Neural modeling and imaging of the cortical interactions underlying syllable production. Brain and Language, 96(3), pp. 280-

Guenther, F.H. (2016). Neural Control of Speech. Cambridge, MA: MIT Press.

Hagedorn, C., Proctor, M., Goldstein, L., Wilson, S., Miller, B., Gorno-Tempini, M., \& Narayanan, S. (2017). Characterizing Articulation in Apraxic Speech Using Real-time Magnetic Resonance Imaging. Journal of Speech, Language and Hearing Research, 60,

914 Hardcastle, W. J. (1985). Some phonetic and syntactic constraints on lingual coarticulation during /k1/ sequences. Speech Communication, 4, 247-263.

916 Hickok, G. (2017). Computational neuroanatomy of speech production. Nature Reviews $917 \quad$ Neuroscience, 13(2), 135-145.

918 Hindson, B. A., \& Byrne, B. (1997). The status of final consonant clusters in English syllables: 
Evidence from children. Journal of Experimental Child Psychology, 64(1), 119-136.

920 Keating, P., Wright, R., \& Zhang, J. (1999). Word-level asymmetries in consonant articulation. UCLA Working Papers in Phonetics, 97, 157-173

922 Klapp, S.T. (1995). Motor response programming during simple and choice reaction time: The role of practice. Journal of Experimental Psychology: Human Perception and

925 Performance, 21(5), 1015-1027.

Klapp, S.T. (2003). Reaction Time Analysis of Two Types of Motor Preparation for Speech Articulation: Action as a Sequence of Chunks, Journal of Motor Behavior, 35:2, 135-150.

Kuhl, P. K., Ramirez, R., Bosseler, A., Lin, J.-F., \& Imada, T. (2014). Infants’ brain responses to speech suggest Analysis by Synthesis. Proceedings of the National Academy of Sciences, $111,11238-11245$.

Lashley, K.S. (1951). The problem of serial order in behavior. In Jeffress, L.A., editor, Cerebral Mechanisms in Behavior. Wiley, New York.

Lee, O., \& Redford, M.A. (2015). Verbal and spatial working memory load have similarly minimal effects on speech production. Proceedings of the 18th International Congress of Phonetic Sciences. Glasgow, UK: The University of Glasgow. ISBN 978-0-85261-941-4.

Levelt, W. J. M. (1989). ACL-MIT Press series in natural-language processing. Speaking: From intention to articulation. Cambridge, MA, US: The MIT Press.

Levelt, W.J.M., \& Wheeldon, L. (1994). Do speakers have access to a mental syllabary? Cognition, 50, 239-269.

Levelt, W.J.M., Roelofs, A., \& Meyer, A.S. (1999). A theory of lexical access in speech production. Behavioral and Brain Sciences. 22, 1-75.

Loevenbruck, H., Collins, M.J., Beckman, M.E., Krishnamurth, A.K., \& Ahalt, S.C. (1999). 
Temporal coordination of articulatory gestures in consonant clusters and sequences of consonants. In O. Fujimura, B.D. Joseph, \& B. Palek (Eds.) Proceedings of Linguistics Phonetics (pp. 547-573). Prague: The Karolinum Press.

945 MacKay, D.G. (1970). The structure of words and syllables: Evidence from errors in speech. Cognitive Psychology, 3(2), 210-227.

947 MacNeilage, P. (1998). The Frame/Content theory of evolution of speech production. Behavioral and Brain Sciences, 21, 499-511.

949 Marian, V., Blumenfeld, H.K., \& Kaushanskaya, M. (2007). The Language Experience and 950 Proficiency Questionnaire (LEAP-Q): assessing language profiles in bilinguals and multilinguals. Journal of Speech, Language, and Hearing Research, 50(4), 940-967.

952 Mowrey, R.A., MacKay, I.R. (1990). Phonological primitives: electromyographic speech error 953 evidence. Journal of the Acoustical Society of America, 88(3):1299-1312.

954 Pan, S.C., \& Rickard, T.C. (2015). Sleep and motor learning: Is there room for consolidation? Psychological Bulletin, 141(4), 812-834.

956 Pierrehumbert, J. \&, R. Nair (1995). Word games and syllable structure, Language and Speech, $957 \quad 38,78-116$.

958 Rochet-Capellan, A., Richer, L., Ostry, D.J. (2012). Non-homogeneous transfer reveals specificity 959 in speech motor learning. Journal of Neurophysiology, 107(6):1711-1717.

960 Rong, F., Isenberg, A.L., Sun, E., \& Hickok, G. (2018). The neuroanatomy of speech sequencing $961 \quad$ at the syllable level. PLOS ONE, 13(10): e0196381.

962 Segawa, J.A., Tourville, J.A., Beal, D.S., \& Guenther, F.H. (2015). The neural correlates of speech 963 motor sequence learning. Journal of Cognitive Neuroscience, 27(4), 819-831.

964 Segawa, J.A., Masapollo, M., Tong, M., Smith, D.J. \& Guenther, F.H. (2019). Chunking of 
phonological units in speech sequencing. Brain \& Language.

966 Sevald, C.A., Dell, G.S., \& Cole, J.S. (1995). Syllable structure in speech production: are

$967 \quad$ syllables chunks or schemas? Journal of Memory and Language, 34, 807-820.

968 Segawa, J.A. (2013). Neural representations used by the brain regions underlying speech

969 production. Ph.D. thesis, Boston University.

970 Segawa, J.A., Tourville, J.A., Beal, D.S., \& Guenther, F.H. (2015). The neural correlates of speech 971 motor sequence learning. Journal of Cognitive Neuroscience, 27(4), 819-831.

972 Shattuck-Hufnagel, S. (1979). Speech errors as evidence for a serial-ordering mechanism in

973 sentence production. In Cooper, W.E., \& Walker, E.C.T. (Eds.), Sentence processing:

974 psycholinguistic studies presented to Merrill Garrett (pp. 295-342). Hillsdale, NJ:

975 Lawrence Erlbaum.

976 Shattuck-Hufnagel, S. (1983). Sublexical units and suprasegmental structure in speech

977 production planning. In MacNeilage, P.F. (Ed.), The production of speech (pp.109-136).

$978 \quad$ New York: Springer.

979 Shattuck-Hufnagel, S. (1986). The representation of phonological information during speech

980 production planning: Evidence from vowel errors in spontaneous speech. Phonology

$981 \quad$ Yearbook, 3, 117-149.

982 Shattuck-Hufnagel, S. (1992). The role of word structure in segmental serial ordering. Cognition, $983 \quad 42,213-259$.

984 Shattuck-Hufnagel, S. (2015). Prosodic frames in speech production. In Redford, M. (Ed.), The

985 Handbook of Speech Production (pp. 419-444). Hoboken, New Jersey: John Wiley \& Sons

986 Inc.

987 Song, J.Y., Demuth, K., Shattuck-Hufnagel, S., \& Ménard, L. (2013). The effects of coarticulation 
and morphological complexity on the production of English coda clusters: Acoustic and articulatory evidence from 2-year-olds and adults using ultrasound. Journal of Phonetics 41, 281-295.

Stevens, K.N., \& Keyser, S.J. (2010). Quantal theory, enhancement and overlap. Journal of Phonetics, 38(1), 10-19.

Sternberg, S., Monsell, S., Knoll, R. R., \& Wright, C. E. (1978). The latency and duration of rapid movement sequences: Comparisons of speech and typewriting. In G. E. Stelmach (Ed.), Information processing in motor control and learning (pp. 117- 152). New York: Academic Press.

Tiede, M., Shattuck-Hufnagel, S., Johnson, B., Ghosh, S., Matthies, M., Zandipour, M., et al. (2007). Gestural phrasing in /kt/ sequences contrasting within and across word contexts. Proceedings of the 16th International Congress of Phonetic Sciences, Saarbrücken (521524), Germany.

Treiman, R. (1984). On the status of final consonant clusters in English syllables. Journal of Verbal Learning and Verbal Behavior, 23(3), 343-356.

Tremblay, S., Shiller, D.M., \& Ostry, D.J. (2003). Somatosensory basis of speech production. Nature, 423(6942), 866-869.

Tremblay, S., Houle, G., \& Ostry, D.J. (2008). Specificity of speech motor learning. Journal of Neuroscience, 28(1), 2426-2434.

Wagner, R., Torgesen, J., and Rashotte, C. (1999). Comprehensive Test of Phonological Processing. Examiner's Manual. Pearson Assessments: San Antonio, TX.

Walker, M.P., Brakefield, T., Morgan, A., Hobson, J.A., \& Strickgold, R. (2002). Practice with sleep makes perfect: sleep-dependent motor skill learning. Neuron, 425, 616-620. 
1011 Walker, M.P., Brakefield, T., Hobson, J.A., \& Strickgold, R. (2003). Dissociable stages of human 1012 memory consolidation and reconsolidation. Nature, 35, 205-211.

1013 Warker, J.A., \& Dell, G.S. (2015). New phonotactic constraints learned implicitly by producing 1014 syllable strings generalize to the production of new syllables. Journal of Experimental 1015 Psychology: Learning, Memory, and Cognition, 41(6), 1902-1910.

1016 Wilson, C., Davidson, L. \& Martin, S. (2014). Effects of acoustic-phonetic detail on cross1017 language speech production. Journal of Memory and Language 77, 1-24.

1018 Wright, D.L., Robin, D., Rhee, J., Vaculin, A., Jacks, A., Guenther, F.H., \& Fox, P.T. (2009).

1019 Using the self-select paradigm to delineate the nature of speech motor programming. $1020 \quad$ Journal of Speech, Language, and Hearing Research, 52, 755-765. 


\section{Tables and captions}

1022 Table 1: International phonetic alphabet (IPA) transcription and orthography for speech stimuli 1023 used to elicit the syllable-initial (onset) and syllable-final (coda) cluster targets (underlined). Each 1024 column represents the stimuli used in the four different experimental conditions. Each row 1025 represents the training and test stimuli used for each of the two experimental groups (see text for 1026 further explanation).

1027 Table 2: Mean error rates in the test blocks by error subtype, word type, and stimulus item. 


\section{Figures and captions}

1030 Figure 1: Bar plots of the mean performance measures (across words and subjects) from the

1031 training blocks as a function of time (first five trials on day one vs. last five trials on day one vs.

1032 first five trials on day two). (Left panel) Mean percentage of sequencing errors. (Middle panel)

1033 Mean durations of properly sequenced utterances. (Right panel) Mean RTs of properly

1034 sequenced utterances. Error bars represent standard error of the mean. Abbreviations: $m=$ 1035 marginally significant, $*=\operatorname{significant}(p<.05), * *=\operatorname{significant}(p<.01), * * *=\operatorname{significant}(p<$ $1036 \quad .001)$.

1037

1038 Figure 2: Bar plots of the mean sequencing error rates (across word pairs and subjects) for the

1039 first five trials in the test blocks (on day two) as a function of experimental condition (practiced 1040 CCVCC, practiced $O C$, practiced CC, and novel CCVCC) and stimulus item (first word vs. second 1041 word). Error bars represent standard error of the mean. Abbreviations: $m=$ marginally significant, $1042 *=$ significant $(p<.05), * *=$ significant $(p<.01), * * *=$ significant $(p<.001)$.

1044 Figure 3: Bar plots of the mean utterance durations (across word pairs and subjects) for the first 1045 five trials in the test blocks (on day two) as a function of experimental condition (practiced 1046 CCVCC, practiced $O C$, practiced CC, and novel CCVCC) and stimulus item (first word vs. second 1047 word). Error bars represent standard error of the mean. Abbreviations: $m=$ marginally significant, $1048 *=$ significant $(p<.05), * *=$ significant $(p<.01), * * *=$ significant $(p<.001)$.

1050 Figure 4: Bar plots of the mean reaction times and inter-item-intervals (across subjects) for the 1051 first five trials in the test blocks (on day two) plotted as a function of experimental condition 
1052 (practiced CCVCC, practiced OC, practiced CC, and novel CCVCC). Error bars represent

1053 standard error of the mean. Abbreviations: $m=$ marginally significant, $*=$ significant $(p<.05)$,

$1054 * * *$ significant $(p<.01), * * *=$ significant $(p<.001)$.

1055

1056 Figure 5: Bar plots of the mean overall duration of word pairs (i.e., first word duration + inter-

1057 item-interval duration + second word duration) during test trials as a function of experimental

1058 condition (practiced CCVCC vs. practiced OC vs. practiced CC vs. novel CCVCC). Error bars

1059 represent standard error of the mean. Abbreviations: $m=$ marginally significant, $*=$ significant $(p$

$1060<.05), * *=$ significant $(p<.01), * * *=$ significant $(p<.001)$.

1061

1062 Figure 6: Schematic of the GODIVA model's account of sub-syllabic sequencing in the novel 1063 CCVCC, practiced $O C$, and practiced CCVCC conditions. $\mathrm{G}_{\mathrm{x}}=$ articulatory gesture for phoneme

$1064 \mathrm{x}$. The blue squares/circles with bold outlines in the lower tier refer to motor programming units. 

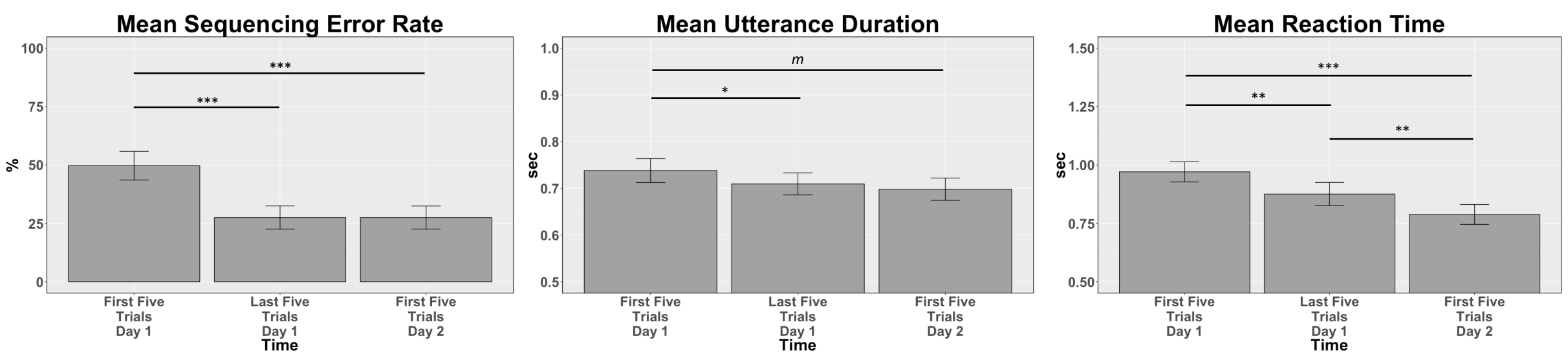


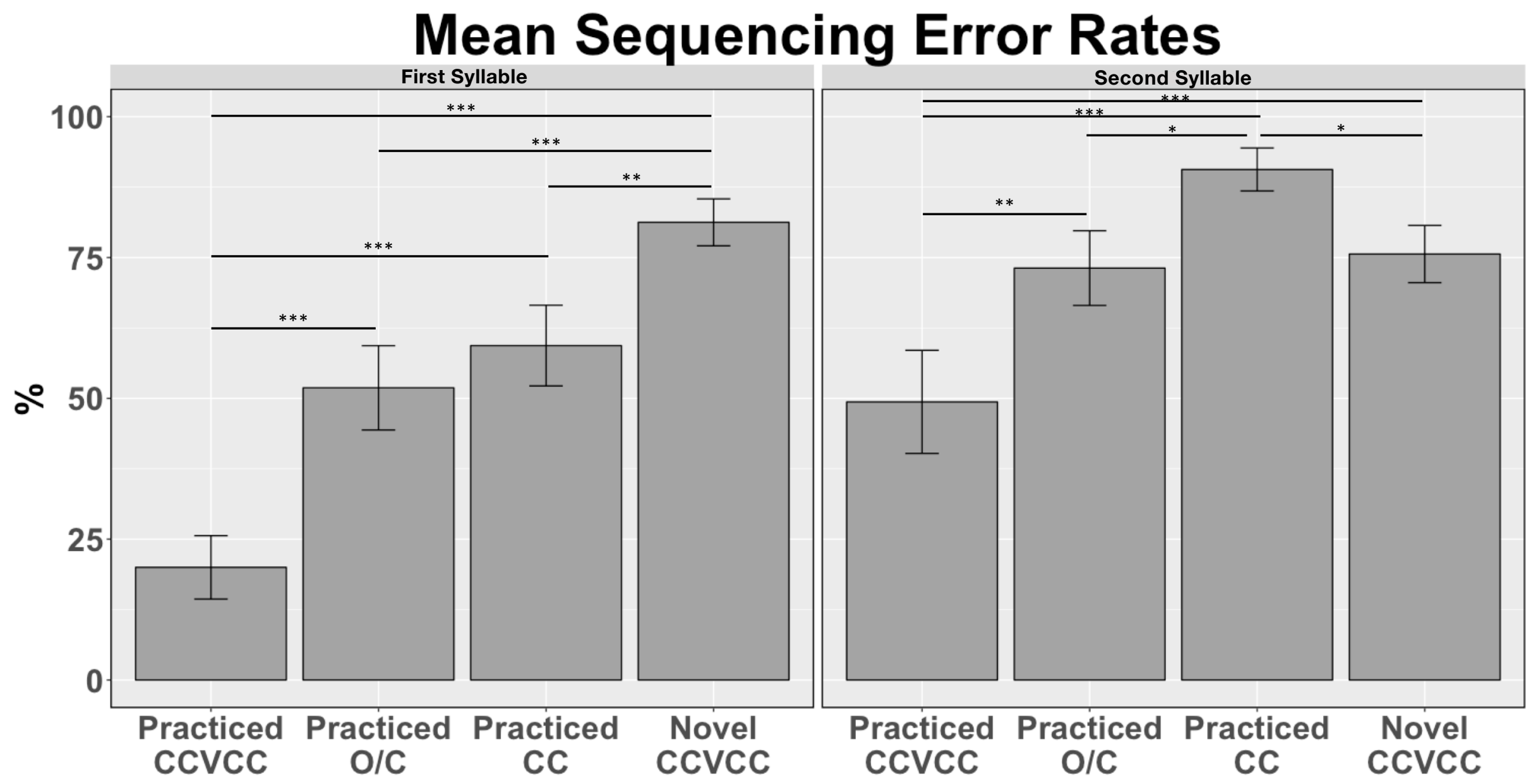




\section{Mean Utterance Duration}

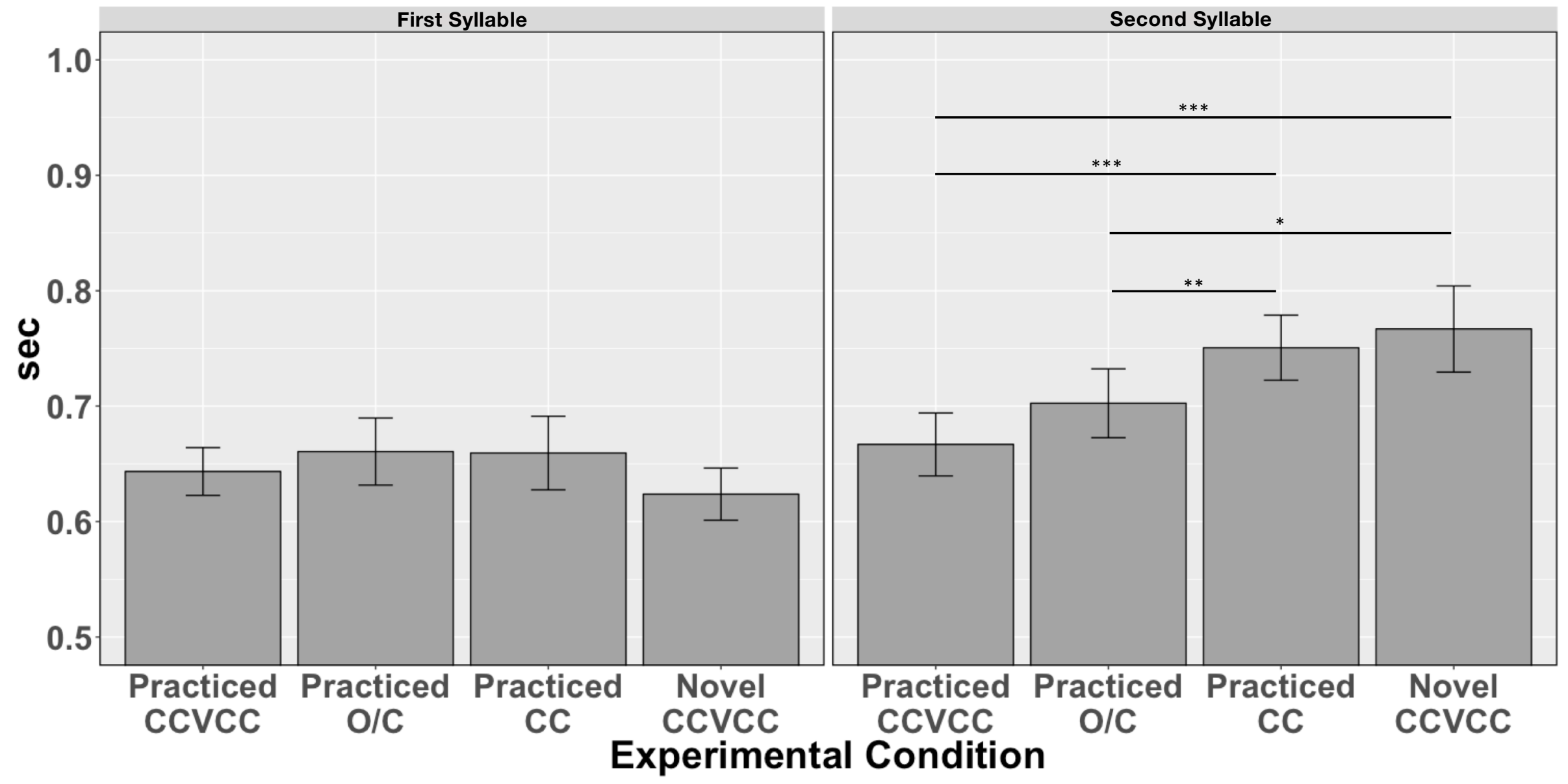



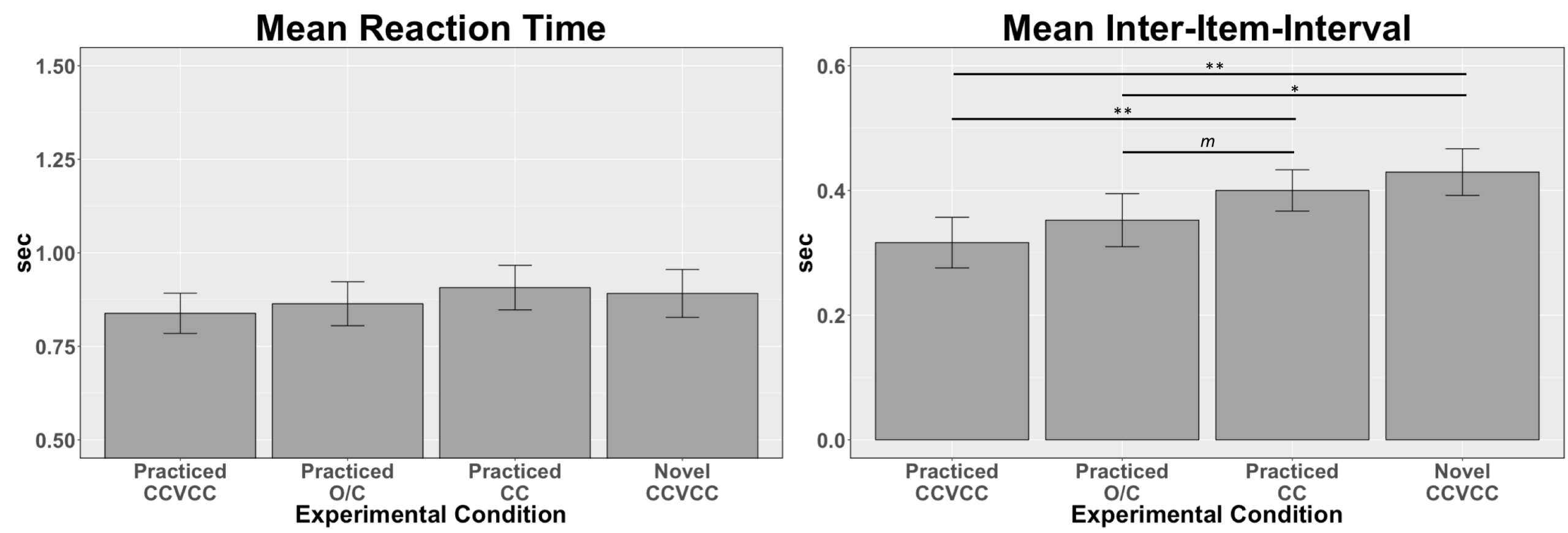




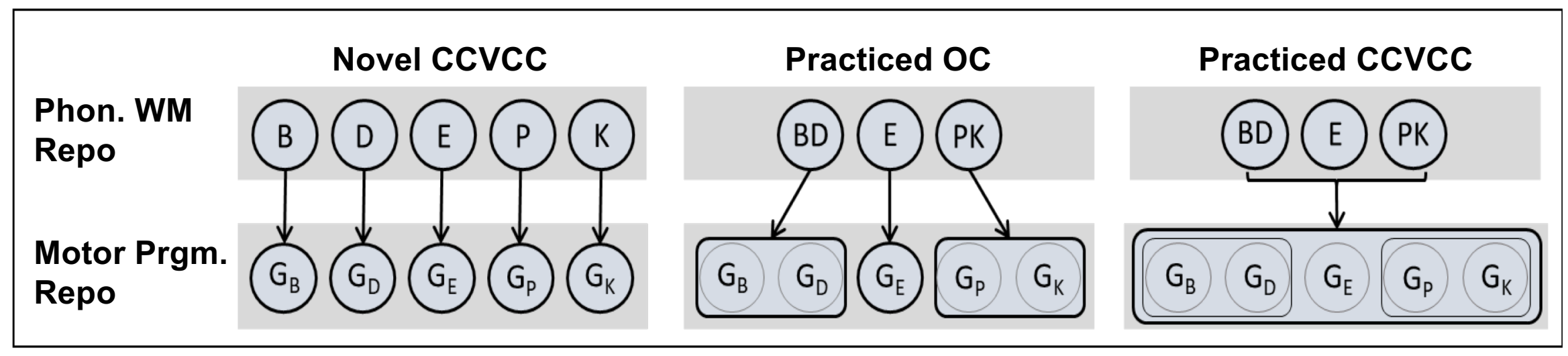




\section{S.1 Time-course information in speech motor-sequence learning}

13 Another common analysis method used to examine the time-course of motor-sequence learning

14 involves comparing average performance (e.g., rate of sequence completion) over some equivalent

15 duration or number of trials within the training phase. This type of calculation would allow

16 researchers to compare performance for each training block relative to the other blocks in order to

17 identify early versus late stages of learning. Figure S1 plots each of our motor performance

18 measures - mean sequencing error rates, utterance duration, and reaction time - across the six

19 training blocks (four on day one and two on day two).

\section{S.1.1. Dissociation between execution of articulatory timing and phonological content}

As figure S1 shows, the overall time-course of improvement of sequencing error rates (top panel) and utterance duration (middle panel) differ. Specifically, considerable improvements in error rates occur very rapidly within the first training block, whereas gains in duration do not emerge until the final block of training on day one. This finding suggests that speakers may learn different

\footnotetext{
* Current address: Department of Speech, Language, and Hearing Sciences, University of Florida, Gainesville, FL 32610
} 
aspects of sequence production on different time scales; the ability to select and generate the proper sequence of articulatory motor gestures (as measured by error rates) appears to start quickly, but

27 the execution of articulatory timing information (as measured by utterance duration) may take

28 longer to establish. Using electromagnetic articulography, ongoing studies are examining gestural

29 coordination in non-native sequence productions more carefully (Cheng, Masapollo, Hagedorn, \&

30 Buchwald, in progress; Masapollo, Kearney, \& Guenther, in progress).

31 The aforementioned finding is also compatible with claims that the execution of

32 articulatory timing and phonological content are mediated by different neural substrates (Bohland

33 et al., 2010; Guenther, 2016). Specifically, it has been proposed that prefrontal medial areas (left

34 pre-supplementary motor area, left supplementary motor area) are recruited in coordinating the

35 speech articulators in time to achieve motor goals in sequence, while lateral areas (left inferior

36 frontal sulcus, left ventral premotor cortex) are thought to be recruited to generate speech motor

37 programs for articulation.

38 S.1.2. Post-delay gains in motor sequence performance

39 As figure $\mathrm{S} 1$ shows, there is a trend for the production timing measures - mean utterance duration

40 and reaction time - to show post-delay gains between the end of training on day one (block 4) and

41 the start of training on day two (block 5). Analogous off-line improvements in performance have

42 been reported in other types of motor-sequence learning studies (albeit using different tasks, e.g.,

43 finger-tapping) and have been hypothesized to depend on sleep-mediated memory consolidation

44 (see, e.g., Walker et al., 2002). However, other have argued that these types of post-delay gains

45 may simply be an artifact of data averaging in the calculation of pre-post gain scores (for

46 discussion, see Brawn et al., 2010; Pan \& Rickard, 2015). For example, fatigue build up might

47 give rise to the worsening of sequence performance at the end of a long training session, which in 
turn, might affect pre-post gain difference scores. In the present case, however, the time-course

49 plots suggest that there is no performance fatigue worsening performance between the end of

50 training on day one, consistent with the hypothesis that sleep may help to enhance some aspects

51 of motor performance. Ongoing work in our laboratory is testing the hypothesis that sleep

52 quality/duration plays an important role in encoding newly acquired speech motor sequences into

53 long-term procedural memory. Specifically, we are examining whether sequence performance

54 following sleep is better than performance after an equivalent period of time during wakefulness.

\section{S.1.3. Incomplete motor learning}

56 Figure S1 (top panel) indicates that, by the end of training, the mean sequencing errors rates are

57 roughly 25 percent, which is still relatively high. Additionally, Figure 3 (in the main text) indicates

58 that the average practiced $C C V C C$ word duration is roughly $650 \mathrm{msec}$. Both of these findings

59 suggest incomplete motor learning, assuming the average adult speech rate of four syllables/second

60 (Kent, 2000; Guenther, 2016). However, we are not making the claim that, following two days of

61 speech motor practice, speakers execute the practiced $C C V C C$ words in a manner that is identical

62 to native-language speech sequences. Rather, the present study captures an early aspect of the

63 learning process (analogous to the earliest stages of second language learning in adulthood) and

64 the extent to which those early performance gains generalize to novel, transfer words.

65 S.2 Effects of syllable position on speech motor-sequence learning

66 We conducted an additional analysis to test for potential effects of syllable position (syllable-initial

67 [onset] clusters vs. syllable-final [coda] clusters) as such effects are suggested by some theories of 68 speech production, especially Articulatory Phonology (see, e.g., Browman \& Goldstein, 1988, 69 1995; Byrd, 1995, 1996; Keating, Wright, \& Zhang, 1999; Byrd, Lee, Riggs, \& Adams, 2005;

70 Bohland, Bullock \& Guenther, 2010). Specifically, we examined the time course of improvement 
71 in the mean sequencing error rates for onset and coda clusters over the two days of speech motor

72 practice. We performed an analysis of variance (ANOVA) with time (five five trials on day one

73 vs. last five trials on day one vs. first five trials on day two) and syllable position (onset clusters

74 vs. coda clusters) as within-subjects factors. The results (shown in Figure S2) revealed a

75 significant main effect of time $\left[F(2,60)=8.523, p<.001, \eta^{2} p=.221\right]$ as well as an interaction

76 effect involving syllable position $\left[F(2,60)=14.477, p<.001, \eta^{2} p=.325\right]$. The effect of syllable

77 position was not significant $\left[F(1,30)=.515, p=.478, \eta^{2} p=.017\right]$.

Simple effects tests on the time $\mathrm{X}$ syllable position interaction revealed that there was an

79 effect of time on sequencing error rates for codas clusters $\left[F(2,30)=14.397, p<.001, \eta^{2}{ }_{p}=.490\right]$,

80 but not onset clusters $\left[F(2,30)=2.495, p=.099, \eta^{2} p=.143\right]$. Follow-up $t$-tests on the pairwise

81 comparisons for coda clusters indicated that subjects produced fewer sequencing errors between

82 the first five $[M=35.0 ; S D=37.2]$ and last five trials $[M=12.0 ; S D=17.5]$ on day one $[t(15)=$

$833.789, p=.002, d=1.00$ ], and between the first five trials on day one and the first five trials on

84 day two $[M=9.0 ; S D=14.8 ; t(15)=5.566, p<.001, d=1.18]$. The difference in mean error rates

85 between the last five trials on day one and the first five trials on day two was not significant $[t(15)$

$86=.618, p=.546, d=.18]$. Although the factors that influence this effect of syllable position are

87 not entirely clear, we hypothesize that speakers may focus their attention on articulating early list

88 versus late list motor elements in a planned speech sequence. 


\section{Supplementary References}

90

91

92

93

94

95

96

97

98

99

100

101

102

103

104

105

106

107

108

109

110

111

112

113

114

115

Bohland, J.W., Bullock, D., \& Guenther, F.H. (2010). Neural representations and mechanisms for the performance of simple speech sequences. Journal of Cognitive Neuroscience, 22(7), pp. 1504-1529.

Brawn, T.P., Fenn, K.M., Nusbaum, H.C., \& Margoliash, D. (2010). Consolidating the effects of waking and sleep on motor-sequence learning. Journal of Neuroscience, 30(42), 1397713982.

Browman, C. \& Goldstein, L. (1988). Some Notes on Syllable Structure in Articulatory Phonology. Phonetica, 45, 140-155.

Browman, C., \& Goldstein, L. (1995). "Gestural syllable position effects in American English," in Producing Speech: Contemporary Issues. For Katherine Safford Harris, edited by F. Bell-Berti and L. Raphael AIP, Woodbury, NY, pp. 19-34.

Byrd, D. (1995). C-Centers Revisited. Phonetica, 52, 285-306.

Byrd, D. (1996). Influences on articulatory timing in consonant sequences. Journal of Phonetics, 24, 209-244.

Byrd., D., Lee, S., Riggs, D., Adams, J. (2005). Interacting effects of syllable and phrase position on consonant articulation. Journal of the Acoustical Society of America, 118(6), 38603873.

Guenther, F.H. (2016). Neural Control of Speech. Cambridge, MA: MIT Press.

Keating, P., Wright, R., \& Zhang, J. (1999). Word-level asymmetries in consonant articulation. UCLA Working Papers in Phonetics, 97, 157-173.

Kent, R. D. (2000). Research on speech motor control and its disorders: a review and prospective. Journal of Communication Disorders, 33(5), 391-427.

Walker, M.P., Brakefield, T., Morgan, A., Hobson, J.A., \& Strickgold, R. (2002). Practice with sleep makes perfect: sleep-dependent motor skill learning. Neuron, 425, 616-620. 

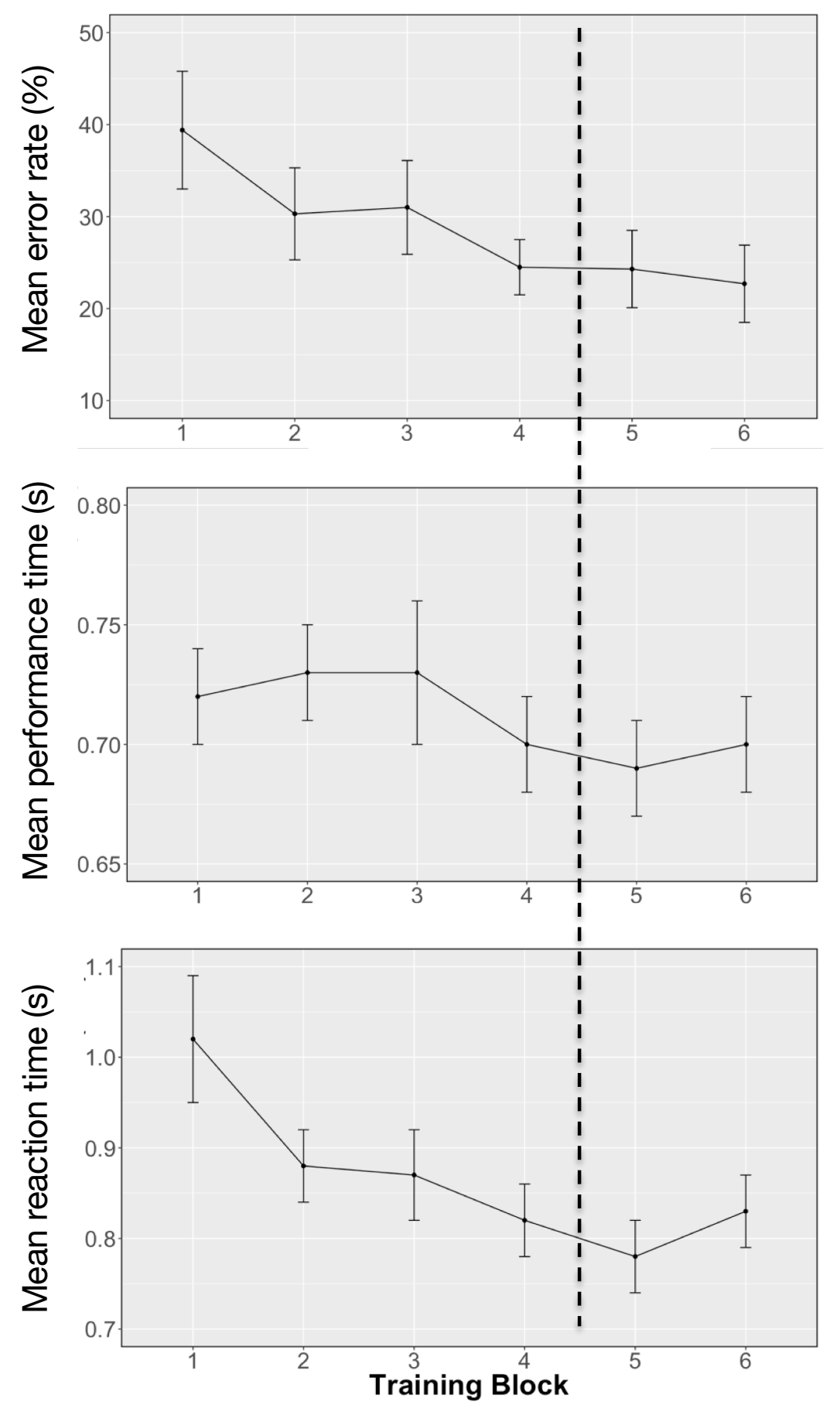

118 Figure S1. Motor performance measures across training blocks. Top panel: Mean percent sequencing error rates. 119 Middle panel: Mean utterance duration ( $\mathrm{s}$; for properly sequenced productions only). Bottom panel: Mean reaction 120 time (s; for properly sequenced productions only). Each data point represents the mean performance measure 121 (averaged across words and subjects) for each training block (10 repetitions/training word). Error bars represent 122 standard errors of the mean. The vertical dashed black line divides the first four training blocks performed on day one 123 from the last two training blocks performed on day two. 


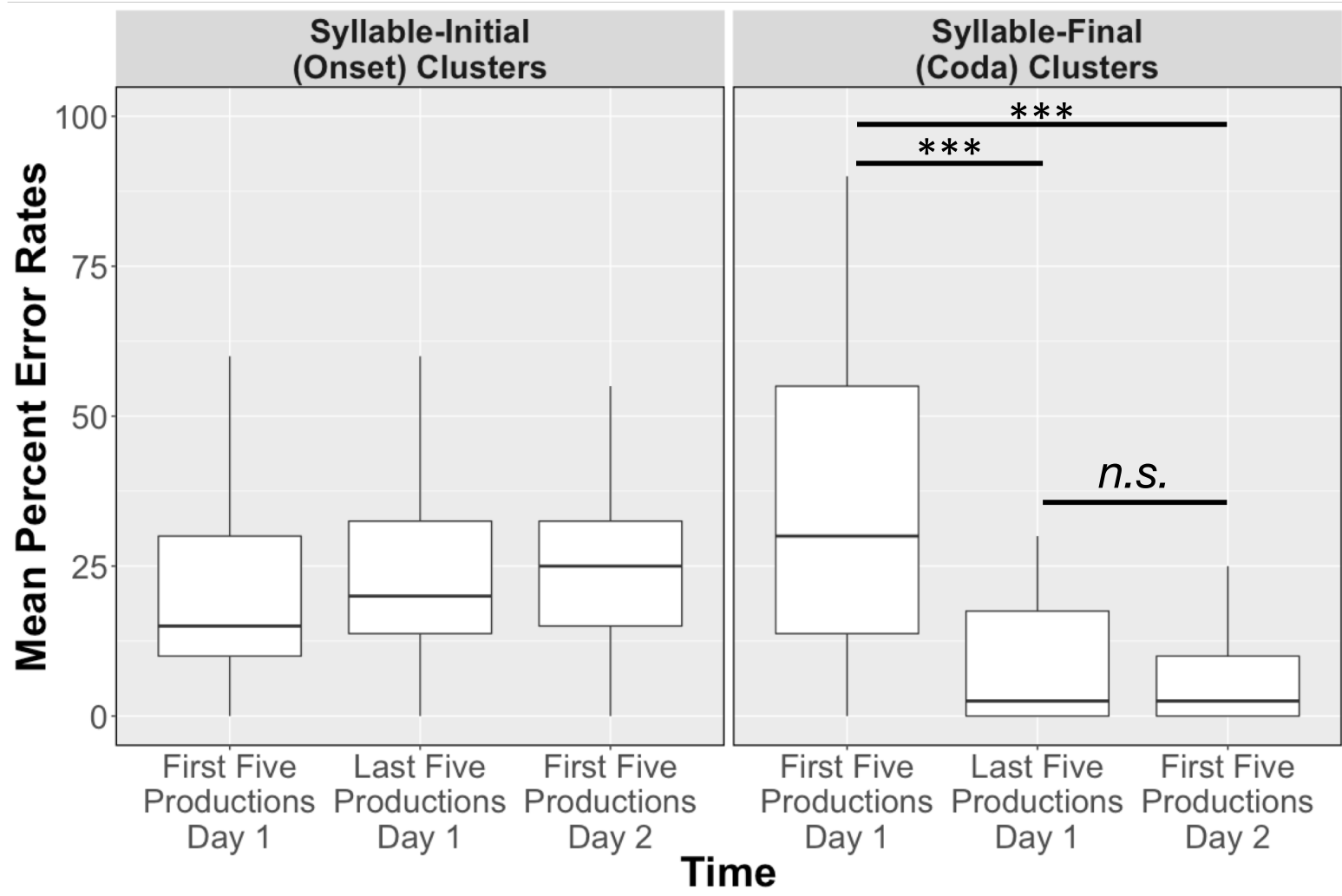

Figure S2. Boxplots of sequencing error rates as a function of syllable position (syllable-initial [onset] clusters vs. syllable-final [coda] clusters) and epoch in training phase (first five productions on day one vs. last five productions 127 on day one vs. first five productions on day two). $* * *=p<.01$. 